\title{
OPEN Pharmacological targeting of host chaperones protects from pertussis toxin in vitro and in vivo
}

\author{
Katharina Ernst ${ }^{1,5}$, Ann-Katrin Mittler ${ }^{1,5}$, Veronika Winkelmann ${ }^{2}$, Carolin Kling ${ }^{1}$, \\ Nina Eberhardt ${ }^{1}$, Anna Anastasia ${ }^{1}$, Michael Sonnabend ${ }^{1}$, Robin Lochbaum ${ }^{2}$, Jan Wirsching ${ }^{1}$, \\ Moona Sakari ${ }^{3}$, Arto T. Pulliainen ${ }^{3}$, Ciaran Skerry ${ }^{4}$, Nicholas H. Carbonetti ${ }^{4}$, Manfred Frick ${ }^{2}$ \& \\ Holger Barth ${ }^{1 \bowtie}$
}

Whooping cough is caused by Bordetella pertussis that releases pertussis toxin (PT) which comprises enzyme A-subunit PTS1 and binding/transport B-subunit. After receptor-mediated endocytosis, PT reaches the endoplasmic reticulum from where unfolded PTS1 is transported to the cytosol. PTS1 ADP-ribosylates G-protein $\alpha$-subunits resulting in increased cAMP signaling. Here, a role of target cell chaperones Hsp90, Hsp70, cyclophilins and FK506-binding proteins for cytosolic PTS1-uptake is demonstrated. PTS1 specifically and directly interacts with chaperones in vitro and in cells. Specific pharmacological chaperone inhibition protects $\mathrm{CHO}-\mathrm{K} 1$, human primary airway basal cells and a fully differentiated airway epithelium from PT-intoxication by reducing intracellular PTS1-amounts without affecting cell binding or enzyme activity. PT is internalized by human airway epithelium secretory but not ciliated cells and leads to increase of apical surface liquid. Cyclophilin-inhibitors reduced leukocytosis in infant mouse model of pertussis, indicating their promising potential for developing novel therapeutic strategies against whooping cough.

Whooping cough is a highly transmissible respiratory disease caused by droplet infection of the respiratory tract with Bordetella (B.) pertussis. This bacterium mediates disease through several virulence factors including adhesion factors and the enzymatically active pertussis toxin $(\mathrm{PT})^{1}$. Characteristic symptoms include severe paroxysmal coughing, which typically lasts for several weeks and can cause secondary complications like vomiting, rib fractures and pneumothorax. In severe cases, further complications such as pneumonia, encephalopathy, seizures and apnea can lead to death especially in newborns and infants ${ }^{2}$. Leukocytosis, the rapid, unregulated expansion of circulating leukocytes, is a hallmark of severe infant disease ${ }^{3,4}$. High levels of leukocytosis are associated with poor disease outcome and death ${ }^{3}$. In 2008, whooping cough caused death in 195,000 of an estimated 16 million cases worldwide according to the world health organization (WHO $)^{5}$. Despite widespread vaccine coverage, disease incidence is at its highest since the $1950 \mathrm{~s}^{6}$.

B. pertussis infects humans and colonizes the ciliated epithelium of the respiratory tract. Adhesion factors like filamentous hemagglutinin (FHA) and fimbria are required for efficient infection. Moreover, B. pertussis produces several toxins that directly influence physiological functions. The precise role of PT for the pathogenesis of $B$. pertussis is still not completely elucidated. In mouse models, PT causes exacerbated and prolonged airway inflammation ${ }^{7}$. Mutant strains of $B$. pertussis that do not express PT, do not cause severe symptoms such as leukocytosis or death in animal models ${ }^{8}$. This observation clearly indicates that PT promotes disease severity and represents an attractive drug target ${ }^{7,9,10}$.

$\mathrm{PT}$ is an $\mathrm{AB}_{5}$-type protein toxin, consisting of one enzyme subunit, the A protomer PTS1, and four different binding (B) subunits PTS2, PTS3, PTS4 and PTS5 occurring in the ratio 1:1:2:1, respectively ${ }^{11,12}$. In B. pertussis, PTS1 and the B pentamer are assembled in a non-covalent manner as PT holotoxin in the periplasm prior to secretion by type IV secretion system ${ }^{11,13}$. The B oligomer facilitates binding of PT to sialic acid-containing glycoproteins present on most mammalian cell types. PT does not appear to bind a single unique receptor, instead it binds in a non-saturable, non-specific manner ${ }^{14}$. PT is endocytosed and follows a retrograde route of intracellular

\footnotetext{
${ }^{1}$ Institute of Pharmacology and Toxicology, University of Ulm Medical Center, Ulm, Germany. ${ }^{2}$ Institute of General Physiology, University of Ulm, Ulm, Germany. ${ }^{3}$ Institute of Biomedicine, Research Unit for Infection and Immunity, University of Turku, Turku, Finland. " Department of Microbiology and Immunology, University of Maryland School of Medicine, Baltimore, MD, USA. ${ }^{5}$ These authors contributed equally: Katharina Ernst and Ann-Katrin Mittler. ${ }^{\square}$ email: katharina.ernst@uni-ulm.de; holger.barth@uni-ulm.de
} 
trafficking travelling through the Golgi apparatus to the endoplasmic reticulum (ER). Consequently, brefeldin A (BFA), which leads to disassembly of the Golgi apparatus, inhibits intoxication of CHO cells with $\mathrm{PT}^{15-17}$. In the ER, binding of ATP to PT destabilizes interaction between PTS1 and B oligomer, which contributes to the release of PTS1 ${ }^{18,19}$. ATP-mediated release occurs in the ER because it is one of the few cellular compartments besides the cytosol and lysosomes/lysosome-related organelles that contains ATP ${ }^{20}$. Released PTS1 is thermally unstable and therefore in its unfolded conformation, which makes it susceptible for transport from the ER into the cytosol via the ER-associated degradation (ERAD) pathway ${ }^{21,22}$. ERAD is responsible for transport of misfolded proteins from the ER into the cytosol where they become ubiquitinated and degraded by the proteasome system $^{22}$. PTS1 is able to utilize this transport pathway and simultaneously evade subsequent degradation since it contains no lysine residues, which are required for ubiquitination ${ }^{23}$. The molecular mechanism underlying membrane transport and refolding of PTS1 and a possible role of host cell factors in this process are not sufficiently understood. In the cytosol, PTS1 covalently transfers ADP-ribose from $\mathrm{NAD}^{+}$onto the a-subunit of trimeric inhibitory $\mathrm{G}$ proteins $(\mathrm{Gi} \alpha)^{24,25}$. This ADP-ribosylation inhibits the activity of Gia as a negative regulator of membrane-bound adenylate cyclase, hence leading to increased intracellular cAMP levels and therefore disturbed signal transduction.

Consequences of cAMP increase depend on the target cells. Presence of the PT-receptor on a wide range of different cell types might explain the various effects of PT during infection with $B$. pertussis. PT inhibits recruitment of neutrophils, monocytes and lymphocytes to the respiratory tract up to 1 week after infection ${ }^{26,27}$. PT leads to decreased amounts of pro-inflammatory chemokines and cytokines and increased bacterial burden in early stages of infection in a mouse model ${ }^{28}$. The current therapeutic regimen only comprises antibiotic treatment, which is important to inhibit rapid spreading of disease via droplet infection but impacts course of disease only if administered in very early stages of infection. Moreover, antibiotic treatment does not improve symptoms including typical paroxysmal cough and in severe manifestations pneumonia, seizures, encephalopathy, apnea and death ${ }^{2}$. Resurgence of pertussis disease and lack of effective treatments necessitates development of novel therapeutics for treatment of severe whooping cough. The PT-dependent nature of severe disease suggests that targeting PT should be an effective strategy for drug development.

We recently demonstrated that specific members of the cyclophilin (Cyp) family, namely CypA and Cyp40 are involved in uptake of PTS1 into the cytosol of target cells ${ }^{29}$. Cyps are members of the peptidyl prolyl cis/trans isomerases (PPIases), which are protein folding helper enzymes that catalyze the rotation of peptide bonds in proteins, assist chaperones such as heat shock protein (Hsp) 90 in protein folding/refolding and are involved in regulating mitochondrial function and permeability (Cyp40 $)^{30-33}$. Both CypA and Cyp40 directly bound to PTS1 in vitro and application of pharmacological Cyp inhibitors such as cyclosporine A (CsA) protected CHO-K1 cells from intoxication with PT. In the presence of Cyp inhibitor, less PTS1 reached the cytosol and there was less ADP-ribosylated Gia, implicating that Cyps are required for uptake of PTS1 into the cytosol ${ }^{29,34}$.

Here, the role of chaperones and PPIases for the uptake of PT into target cells was investigated and Hsp90, Hsp70, as well as specific Cyps and FK506 binding proteins (FKBPs) were identified as novel interaction partners of PTS1. Application of specific pharmacological inhibitors revealed their crucial functional role for the transport of PTS1 into the cytosol of target cells, protected cells, including primary human airway cells, from intoxication with PT and reduced leukocytosis in an infant mouse model of pertussis.

\section{Results}

Specific pharmacological inhibition of chaperone and PPlase activities protects cells from intoxication with PT. A previously established morphology based assay of cellular intoxication was used to determine the functional role of host cell chaperones and PPIases during PT-intoxication ${ }^{29,35}$. CHO-K1 cells show a characteristic clustering morphology if treated with PT (Fig. 1). CHO-K1 cells were pre-incubated prior to PT-treatment with radicicol (Rad) to block Hsp90-activity, and FK506 or CsA to inhibit FKBP or Cyp activity, respectively. Presence of Rad, CsA or FK506 robustly reduced CHO-K1 cell clustering compared to cells treated with PT (Fig. 1a). BFA was used as an inhibitor of PT-induced CHO-K1 cell clustering ${ }^{17,29,36}$. Additionally, fewer cells were observed in wells treated with PT (Fig. 1a). This effect was also inhibited by Rad, CsA and FK506. Hsp70 inhibitors VER-155008 (VER) and HA9 ${ }^{37}$ both inhibited PT-intoxication (Fig. 1b). The inhibitors alone had no effect on cell morphology or cell number (Supplemental Fig. 1a,b). Taken together, the results indicate a functional role of Hsp90, Hsp70, Cyps and FKBPs for PT-intoxication.

Inhibition of chaperone/PPlase activities leads to reduced ADP-ribosylated Gi $\alpha$ without interfering with enzyme activity of PTS1 or binding of PT to cells. After incubation of CHO-K1 cells with PT in the presence or absence of the chaperone and PPIase inhibitors, cells were lysed and incubated with fresh PTS1 in the presence of biotin-labeled NAD ${ }^{+}$. During this incubation, the portion of Gia, which has not been modified by PTS1 during the previous treatment of living cells with PT, was ADP-ribosylated in vitro ${ }^{29}$. This results in biotin-labeling of Gia due to the covalent transfer of the biotin-labeled ADP-ribose moiety from $\mathrm{NAD}^{+}$. Biotin-labeled i.e. ADP-ribosylated Gia was then detected by Western blotting. A strong signal was obtained from control samples, whereas a weaker signal was observed in the presence of PT, showing that in PT-treated cells a substantial amount of Gia was already ADP-ribosylated and could not serve as substrate in the subsequent in vitro ADP-ribosylation reaction. Pre-treatment of cells with chaperone/PPIase inhibitors reduced the ADP-ribosylation of Gia compared to cells treated with PT alone (Fig. 2a). Treatment of cells with the inhibitors only did not affect the subsequent in vitro ADP-ribosylation of Gia by PTS1 (Supplemental Fig. 1c).

Inhibition of Cyps and FKBPs prevented PT-mediated cAMP increase in the iGIST bioassay (Fig. 2b). This assay is based on HEK293 cells that express the Gia-coupled somatostatin receptor 2 (SSTR2) as well as a luminescent cAMP probe ${ }^{38}$. Control samples treated with forskolin to stimulate adenylate cyclase activity and 
a

con

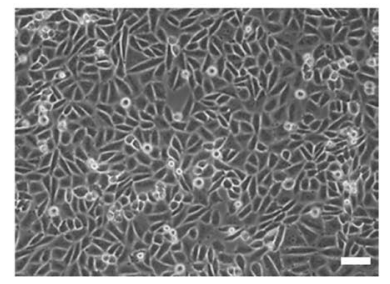

$\mathrm{PT}+\mathrm{Rad}$

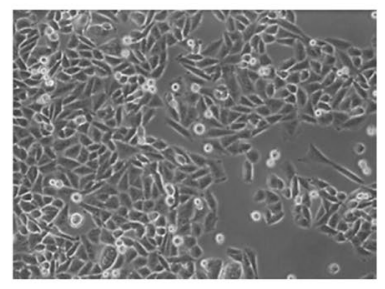

b

con

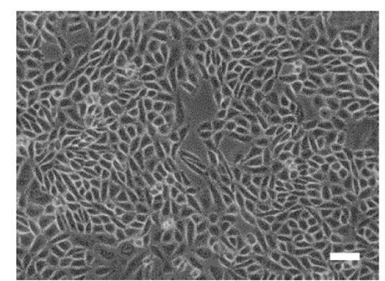

$P T+V E R$

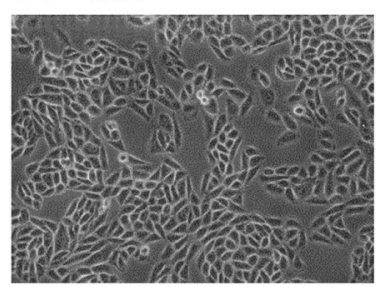

PT

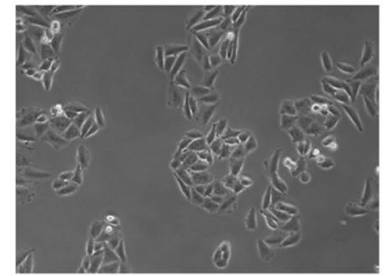

$\mathrm{PT}+\mathrm{CsA}$

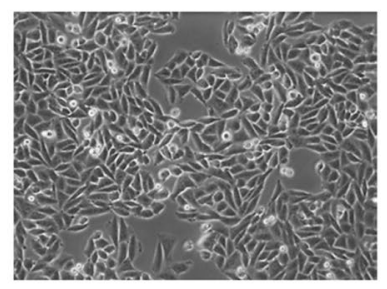

$\mathrm{PT}+\mathrm{BFA}$

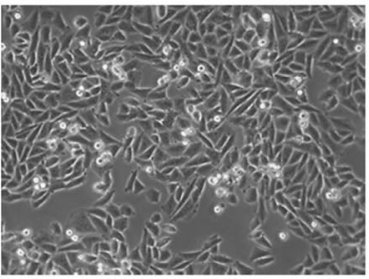

$\mathrm{PT}+\mathrm{FK} 506$

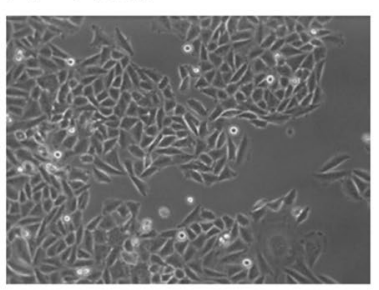

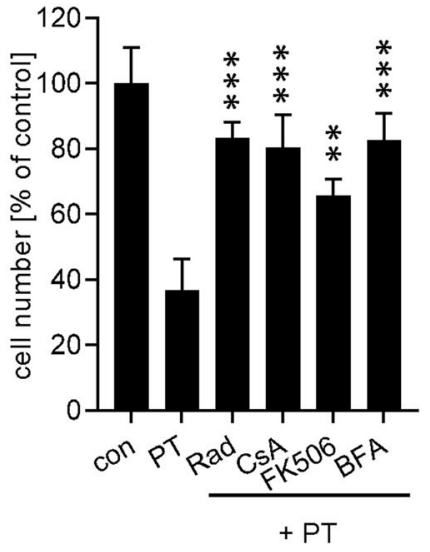

Figure 1. (a) Effect of BFA, Rad, FK506 and CsA on the intoxication of CHO-K1 cells with PT. CHO-K1 cells were pre-incubated with $10 \mu \mathrm{M}$ BFA, Rad, FK506 or CsA or left untreated for control. After $30 \mathrm{~min} 10 \mathrm{ng} / \mathrm{ml} \mathrm{PT}$ were added. $1.5 \mathrm{~h}$ later the culture medium was removed, and cells were further incubated at $37{ }^{\circ} \mathrm{C}$ and $5 \% \mathrm{CO}_{2}$ in fresh medium that did not contain PT or any inhibitor. Pictures were taken after $18 \mathrm{~h}$. A quantitative analysis of total cell number of $\mathrm{CHO}-\mathrm{K} 1$ cells is shown, values are normalized on control cells $(\mathrm{n}=3$, mean \pm SD). (b) Pharmacological inhibition of Hsp70 activity protects cells from PT-intoxication. CHO-K1 cells were treated with VER $(30 \mu \mathrm{M})$ or HA9 $(20 \mu \mathrm{M})$ for $30 \mathrm{~min}$ and then intoxicated with PT $(10 \mathrm{ng} / \mathrm{ml})$ for $18 \mathrm{~h}$. Pictures were taken, and cell numbers were determined as described in A. Significance was tested by one-way ANOVA with Dunnett's multiple comparisons test and refers to samples treated with PT only $\left(^{*} \mathrm{p}<0.05,{ }^{* *} \mathrm{p}<0.01\right.$, $\left.{ }^{* * *} \mathrm{p}<0.001\right)$. Scale bar $=50 \mu \mathrm{m}$.

octreotide to activate SSTR2 which inhibits adenylate cyclase activity show a moderate increase in luminescence signal, i.e. intracellular cAMP (Fig. 2b). In contrast, in samples treated with PT, an enhanced increase in cAMP levels was detected because SSTR2-mediated inhibition of the adenylate cyclase through Gia was impaired. If cells were treated only with forskolin, a comparable increase in cAMP levels was detected with or without PTtreatment (Supplemental Fig. 2b). CsA and FK506 prevented the PT-mediated increase while only marginally affecting cAMP levels in the absence of PT (Fig. 2b). Rad, VER and BFA reduced cAMP levels in the absence of PT (Supplemental Fig. 2a). An inhibitory effect of Rad, VER and BFA on PT-mediated cAMP increase could therefore not be detected with this assay. Solvents of inhibitors had no effect on cAMP levels (Supplemental Fig. 2a).

Taken together, these results suggest that inhibition of chaperones/PPIases reduced PTS1-mediated cytosolic ADP-ribosyltransferase activity. To explain this, we have developed competing hypotheses: (1) the inhibitors reduce the enzyme activity of PTS1 or (2) the inhibitors interfere with the transport of PTS1 into the cytosol.

None of the inhibitors impaired the ADP-ribosylation of Gia by PTS1 in vitro (Fig. 2c, Supplemental Fig. 2c), suggesting that the inhibitors prevent uptake of PTS1 into the cytosol. Binding of PT was not inhibited by 

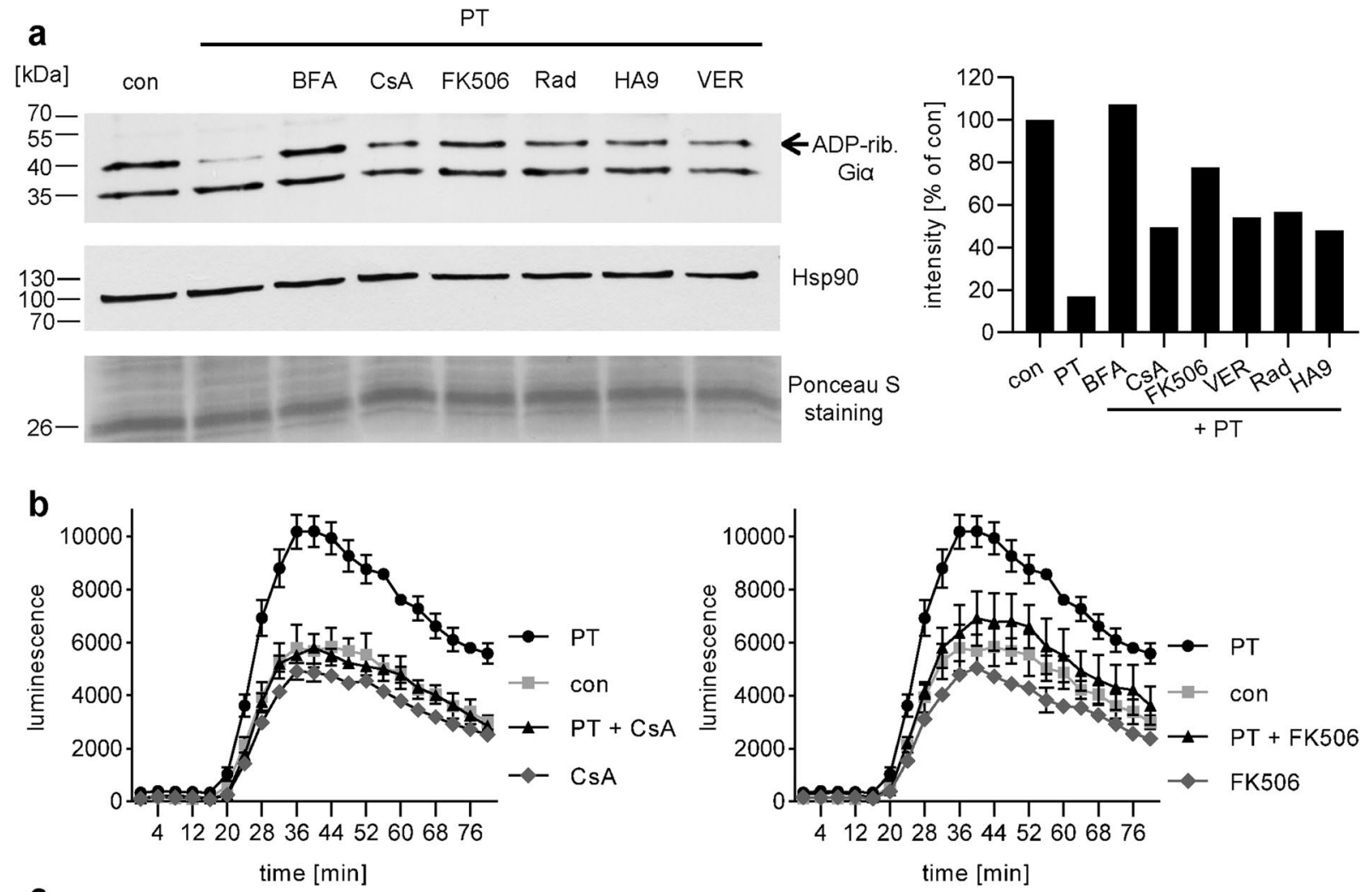

C

$$
\text { time [min] }
$$
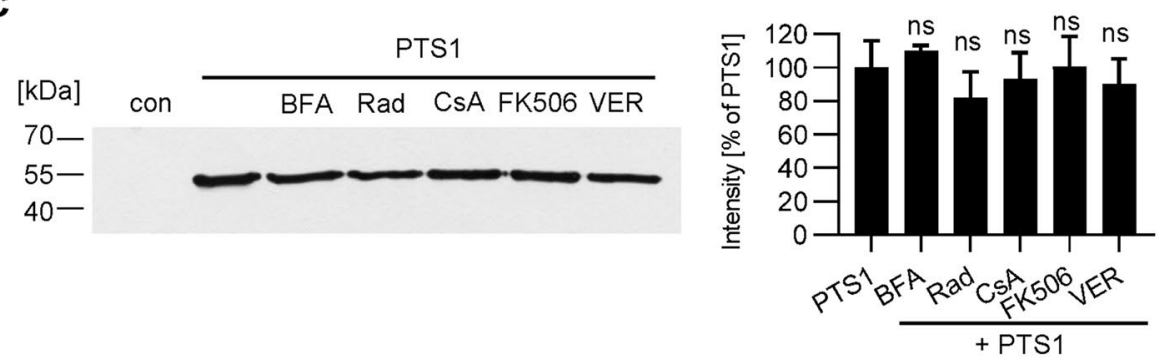

d

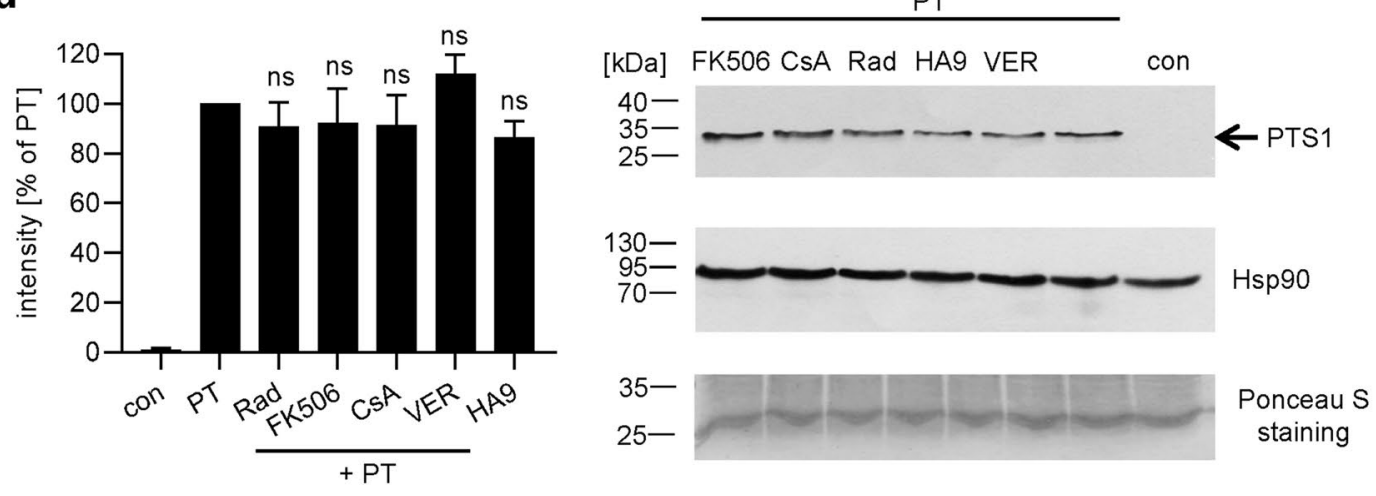


4Figure 2. (a) Effect of Rad, CsA, FK506, VER and HA9 on the ADP-ribosylation status of Gia in PT-treated CHO-K1 cells. Cells were pre-incubated with $20 \mu \mathrm{M}$ of Rad, CsA or FK506, $30 \mu \mathrm{M}$ VER, $100 \mu \mathrm{M}$ HA9 or $20 \mu \mathrm{M}$ BFA for control for $30 \mathrm{~min}$ or left untreated. Then, cells were challenged with $20 \mathrm{ng} / \mathrm{ml} \mathrm{PT}$ for $3 \mathrm{~h}$. Cells were lysed and ADP-ribosylation status of Gia was analyzed. Biotin-labeled (i.e., ADP-ribosylated) Gia was detected. Comparable protein loading was confirmed by Hsp90 and Ponceau S staining. Western blot signals were quantified and normalized to protein loading (Ponceau S). One representative result is shown $(n=3)$. For uncropped blots see Supplemental Fig. S10. (b) Effect of chaperone inhibitors on intracellular cAMP levels in PT-treated cells. iGIST sensor cells (HEK293 cells expressing SSTR2 and luminescent cAMP probe) were incubated with CsA or FK506 $(5 \mu \mathrm{M})$ or left untreated for control for $30 \mathrm{~min}$. $500 \mathrm{ng} / \mathrm{ml}$ PT were added for $3 \mathrm{~h}$ and afterwards inducing medium was added to start the luminescence reaction. A baseline was recorded for $15 \mathrm{~min}$. Then, cells were treated with forskolin to activate adenylate cyclase and with octreotide to activate SSTR2. Luminescence was recorded for $1 \mathrm{~h}$. Values are given as mean $\pm \mathrm{SD}$. Results from one representative experiment are shown. (c) Effect of Rad, CsA, FK506 and VER on enzyme activity in vitro. Recombinant Gia $(0.5 \mu \mathrm{M})$ was pre-incubated with $10 \mu \mathrm{M}$ Rad, CsA, $20 \mu \mathrm{M}$ FK506 or VER for 30 min or with buffer for control. After $30 \mathrm{~min}$ rPTxS1 $(50 \mathrm{nM})$ and $1 \mu \mathrm{M}$ biotin-labeled $\mathrm{NAD}^{+}$were added and incubated for $40 \mathrm{~min}$ at room temperature. Samples were subjected to SDS-PAGE, blotted and ADP-ribosylated Gia was detected with StrepPOD. Western blot signals were quantified. Values are given as percent of samples treated with PTS1 only $(\mathrm{n}=3$ values from 3 independent experiments, mean \pm SD). Significance was tested one-way ANOVA with Dunnett's multiple comparisons test and refers to samples treated with PTS1 only ( $n s$ not significant). For uncropped blot see Supplemental Fig. S11. (d) Influence of Rad, CsA, FK506 and VER on receptor binding of PT to CHO-K1 cells. CHO-K1 cells were pre-incubated with $20 \mu \mathrm{M}$ Rad, CsA, FK506 or $30 \mu \mathrm{M}$ VER or left untreated. After $30 \mathrm{~min}$ cells were cooled to $4{ }^{\circ} \mathrm{C}$ for $15 \mathrm{~min}$. Then $500 \mathrm{ng} / \mathrm{ml} \mathrm{PT}$ were added for $30 \mathrm{~min}$ at $4{ }^{\circ} \mathrm{C}$. After washing, cells were lysed with Laemmli buffer at $95^{\circ} \mathrm{C}$. Cell-bound PT was detected via an anti-PTS1 antibody in Western Blot. Comparable protein loading was confirmed with Hsp90 and Ponceau-S-staining. Quantification of Western blot signals and one representative Western blot is shown. Values were normalized on the amount of loaded protein (Ponceau S) and are given as percent of PT binding (second bar from left). ( $n=4$, mean \pm SEM). Significance was tested by one-way ANOVA with Dunnett's multiple comparisons test and refers to samples treated with PT only (ns not significant). For uncropped blots see Supplemental Fig. S12.

chaperone/PPIase inhibitors (Fig. 2d). This implies that another step of toxin uptake, such as the intracellular membrane transport of PTS1 into the cytosol is the target of these inhibitors.

In the presence of chaperone/PPlase inhibitors, less free PTS1 is detectable in cells. Previously we used fluorescence microscopy to demonstrate that a monoclonal PTS1-antibody recognizes preferably PTS1 when it is detached from the B-subunit pentamer, i.e. cytosolic PTS $1^{29}$. Detection of free PTS1 is decreased following incubation with chaperone/PPIase inhibitors compared to cells challenged with PT only (Fig. 3, Supplemental Fig. 3). This suggests that the inhibitors interfere with uptake of PTS1 into the cytosol. The reason for that might be that endocytosis, retrograde transport to the ER or translocation from the ER to the cytosol are affected. Clustering of cells was still observed in some samples (Rad- and CsA-treated), which might be caused by a small amount of PTS1 that still reached the cytosol in these cells. Notably, the PT-concentration needed for fluorescence experiments is higher than for intoxication experiments in Fig. 1. This might explain why protection by inhibitors against clustering is not as strong as shown in Fig. 1. A reduced PTS1 signal was also observed with BFA, which prevents transport of PT from the Golgi apparatus to the ER and the results are in line with our earlier findings that CsA inhibits uptake of PTS1 into the cytosol ${ }^{29}$. Hence, the reduction of the PTS1 signal in the presence of chaperone/PPIase inhibitors suggests that chaperones/PPIases are involved in uptake of PTS1 to the cytosol.

PTS1 directly and specifically interacts with chaperones and PPIases in vitro. To determine if PTS1 interacts directly with chaperones and PPIases, recombinant chaperones/PPIases were immobilized on a nitrocellulose membrane and overlaid with PTS1. Results in Fig. 4a show specific and concentration-dependent binding of PTS1 to Hsp90, Hsp70 and Hsc70 as well as to CypA, Cyp40, FKBP51 and FKBP52. Previously, we showed that other toxin subunits like the lethal factor of $B$. anthracis or the transport component of $C$. botulinum C2 toxin do not interact with these chaperones/PPIases in the same assay indicating its specificity ${ }^{39,40}$. Ponceau S-staining confirmed transfer of recombinant proteins onto the membrane. Moreover, no interaction was observed with a control protein C3bot from C. botulinum or the small isoform FKBP12. FKBP12 consists of only one PPIase domain. FKBP51 and FKBP52 also contain a PPIase domain and have additional domains that facilitate the interaction with potential client proteins ${ }^{32}$. Here, we investigated the binding of PTS1 to FKBPfragments that only comprise the conserved PPIase domains i.e. FKBP51 FK1 and FKBP52 FK1. Results shown in Fig. $4 \mathrm{~b}$ indicate that there still is an interaction of PTS1 with the fragments, but it is obviously weaker, compared to full-length FKBPs. This suggests that the PPIase domain, as well as the other domains of FKBPs, are involved in binding of PTS1.

Cyp40, FKBP51, Hsp70 and Hsp90 interact with PTS1 in cells for at least 24 h post intoxication. After demonstrating a direct interaction of chaperones/PPIases with PTS1 in vitro and establishing a functional role of these factors during uptake of PT into the cytosol, the duration of the PTS1-Cyp40 interaction was investigated in cells. The proximity ligation assay (PLA) technology encompasses an internal amplification reaction that only occurs if two antibodies against the potential binding partners come into close proxim- 
PT

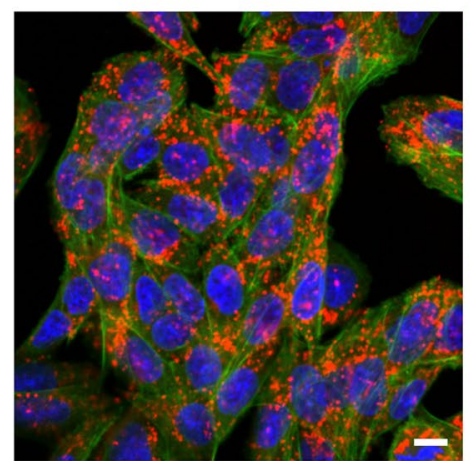

PT + FK506

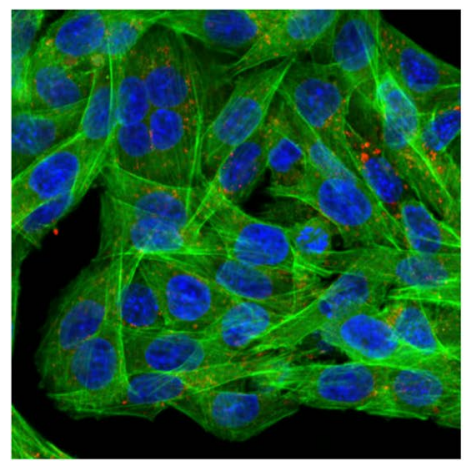

$\mathrm{P} T$ + Rad

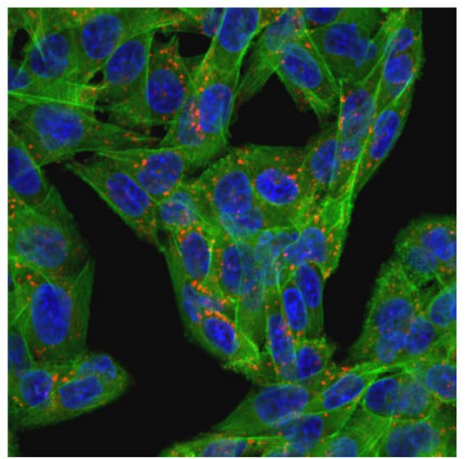

PT + VER

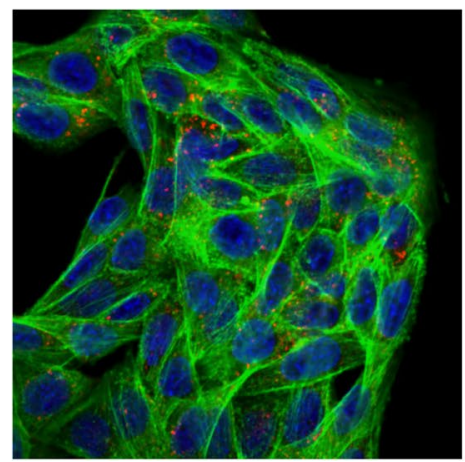

$P T+C s A$

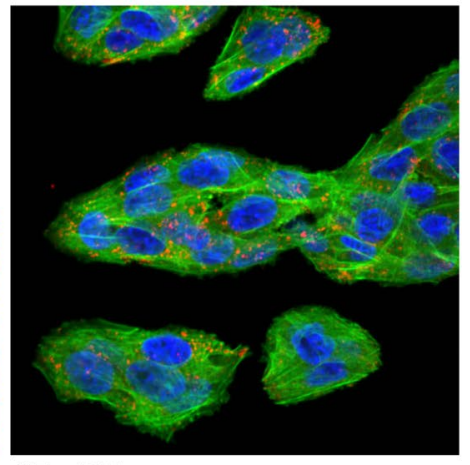

$\mathrm{PT}+\mathrm{BFA}$

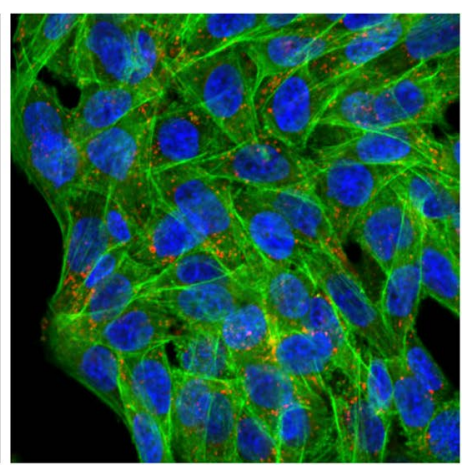

Figure 3. In the presence of inhibitors of Hsp90/Hsp70 and PPIases less free PTS1 is detected in CHO-K1 cells. CHO-K1 cells were pre-incubated with CsA, FK506, Rad $(20 \mu \mathrm{M})$ or VER $(30 \mu \mathrm{M})$ for $30 \mathrm{~min} .20 \mu \mathrm{M}$ BFA were used as control. Then, cells were challenged with $1 \mu \mathrm{g} / \mathrm{ml}$ PT and $1 \mathrm{~h}$ later medium was exchanged. After $24 \mathrm{~h}$, cells were fixed, permeabilized and blocked. Subsequently, cells were probed with an anti-PTS1 antibody, Hoechst and phalloidin-FITC for F-actin staining. Pictures were taken with a Zeiss LSM-710 confocal microscope Red $=$ PTS1, green $=$ F-actin, blue $=$ nucleus, scale bar $=10 \mu \mathrm{m}$.

ity. Every PLA signal detected by fluorescence microscopy represents one interaction event between PTS1 and Cyp40. The interaction between PTS1 and Cyp40 was detected from $30 \mathrm{~min}$ up to $48 \mathrm{~h}$ after incubation of cells with PT (Fig. 4c, Supplemental Fig. 4). CsA or BFA did not inhibit interaction of Cyp40 with PTS1 i.e. the PLA signal, most likely due to high PT concentration (Supplemental Fig. 5). Notably, under these conditions CsA and BFA did not reduce the PTS1 signal per se as shown before with lower concentrations of PT in Fig. 3 (Supplemental Fig. 5). Moreover, interaction between PTS1 and Hsp90, Hsp70 and FKBP51 was demonstrated after $3 \mathrm{~h}$ and $24 \mathrm{~h}$ of PT intoxication (Fig. 4d, Supplemental Fig. 6).

PT invades secretory but not ciliated cells in a human airway epithelium. To determine if the phenomenon of chaperone/PPIase requirement for PT uptake is recapitulated in a model of primary human bronchial airway epithelium, we first demonstrated that PT intoxicates human primary basal cells from airway epithelium in a concentration-dependent manner (Supplemental Fig. 7). Although clustering was not observed in these cells upon PT-treatment, impairment of cell morphology was noted (Supplemental Fig. 7) suggesting some cell damage induced by PT. Application of Rad or CsA reduced ADP-ribosylation of Gia in PT-treated basal cells (Fig. 5a), as analyzed by sequential ADP-ribosylation. Inhibitors alone had no significant effect on ADP-ribosylation of Gia (Supplemental Fig. 8). Mechanistic studies performed in CHO-K1 cells demonstrated that Rad and CsA do not influence enzyme activity or receptor binding but reduce the cytosolic amount of PTS1 in target cells, suggesting that Hsp90 and Cyps are involved in the transport of PTS1 into the cytosol of these cells.

Basal cells that represent one cell type of human bronchial airway epithelial cells (hBAECs) can be differentiated to a functional airway epithelium at air-liquid interface conditions, containing ciliated and secretory cells. By analyzing the effect of PT on the differentiated cell layer by fluorescence microscopy, it became evident that PTS1 was selectively detected in secretory (CC10 or MUC5B positive) cells ${ }^{41}$, but not in ciliated cells ( $\beta$-IV-tubulin positive) (Fig. 5b). Moreover, CsA but not Rad or FK506 reduced the amount of PTS1 detected by fluorescence microscopy in this functional airway epithelium (Fig. 6). Noteworthy, the PTS1-antibody preferentially recognizes PTS1 that is not bound to the B-oligomer, which is most likely located in the cytosol. Rad, CsA or FK506 alone had no adverse effects on morphology of the airway epithelium or tight junctions (Supplemental Fig. 9a). However, VER and BFA revealed strong adverse effects on the airway epithelium showing a reduced cell number for VER-treated cells and nearly no cells were detected after BFA treatment (Supplemental Fig. 9b). 
a

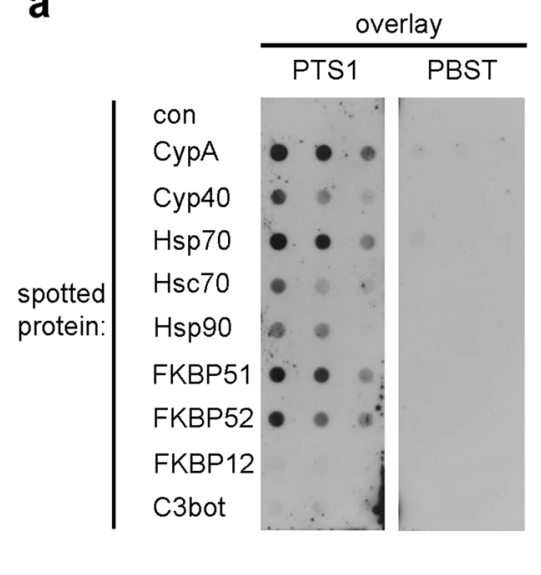

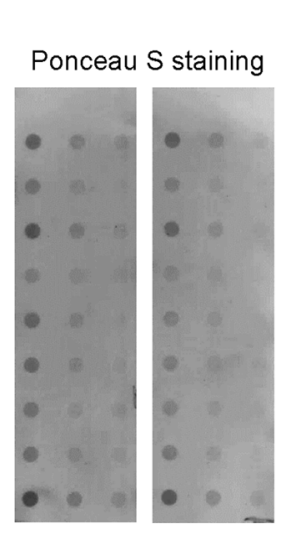

b

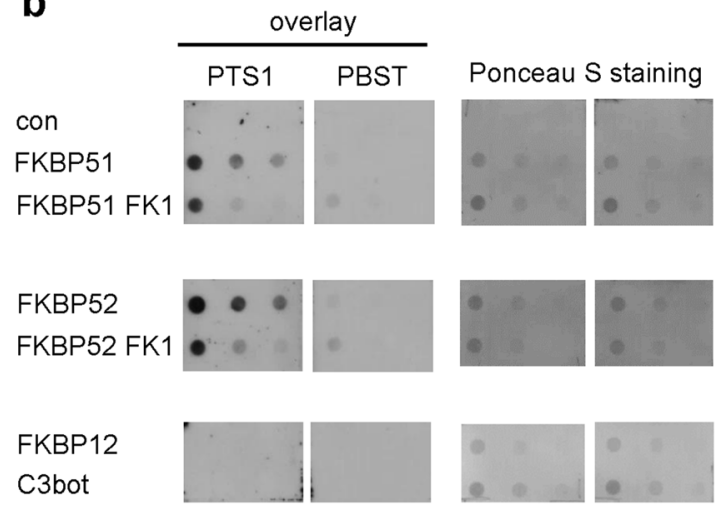

C

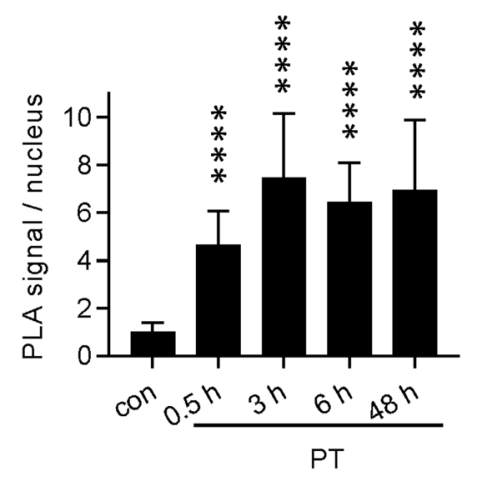

$\operatorname{con} 48 \mathrm{~h}$

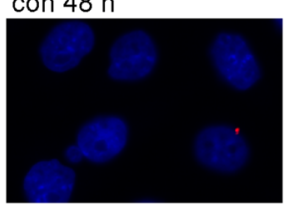

PT $0.5 \mathrm{~h}$

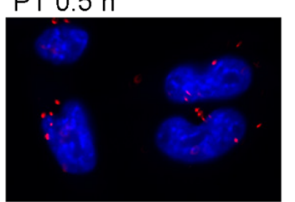

PT 6h

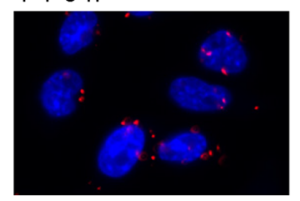

PT $3 \mathrm{~h}$
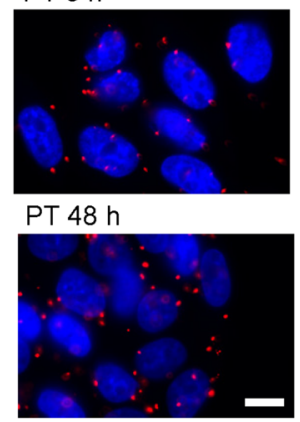

d

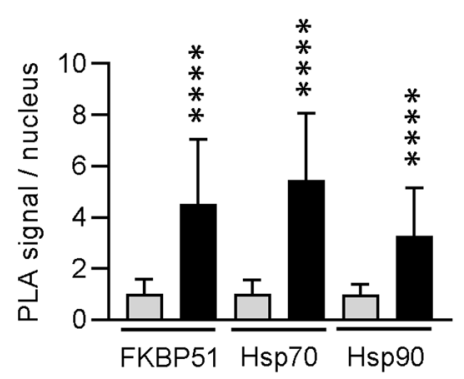

$3 \mathrm{~h}$

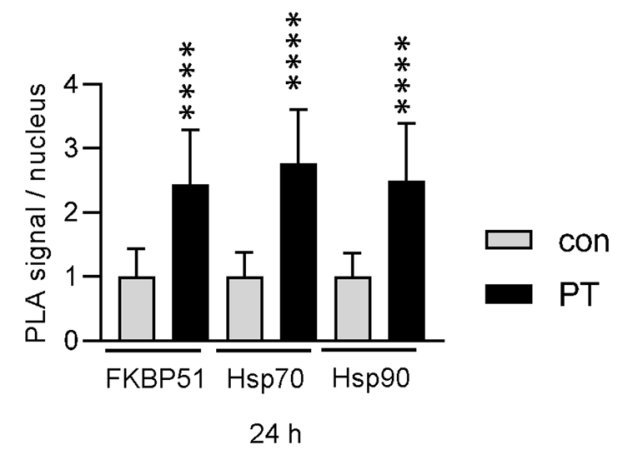

Figure 4. (a) PTS1 interacts with chaperones and PPIases in vitro. Purified chaperones/PPIases were spotted two times onto a nitrocellulose membrane in decreasing concentrations $(1 \mu \mathrm{g}, 0.5 \mu \mathrm{g}, 0.25 \mu \mathrm{g})$. PBS and C3 toxin from C. botulinum were used as negative controls. Membrane was cut and overlay with His-PTS1 (200 ng/ml) or PBST was performed. After extensive washing, bound PTS1 was detected with a specific antibody. PTS1- and PBST-overlayed membranes were detected on the same X-ray film and images were cropped for display purposes only. Comparable amounts of spotted protein were confirmed by Ponceau S-staining. (b) PTS1 binds to the isolated PPIase domains of FKBP51/52. FKBP51/52 and their FK1 fragments i.e. isolated PPIase domains were spotted onto a nitrocellulose membrane. The experiment was further conducted as described in A. All signals were detected on the same X-ray film and images were cropped for display purposes only. (c) Cyp40 interacts with PTS1 in cells. CHO-K1 cells were incubated on ice with PT ( $5 \mu \mathrm{g} / \mathrm{ml}$ ) for 30 min to enable binding or left untreated for control. After washing, the cells were further incubated for $0.5 \mathrm{~h}, 3 \mathrm{~h}, 6 \mathrm{~h}$ and $48 \mathrm{~h}$ at $37^{\circ} \mathrm{C}$. Cells were fixed and fluorescence-based PLA assay was performed according to the manufacturer's manual. PLA signals represent one protein interaction event of PTS1 and Cyp40 and were counted from fluorescence pictures $(n=10$ pictures per condition, mean \pm SD) with ImageJ. Values were normalized to the mean of the control samples. Significance was tested by one-way ANOVA with Dunnett's multiple comparisons test and refers to untreated control samples $\left({ }^{* * *} \mathrm{p}<0.0001\right)$. One representative image is shown for each condition (blue $=$ nucleus, red $=$ PLA signal). Scale bar $=10 \mu \mathrm{m}$. (d) FKBP51, Hsp70 and Hsp90 interact with PTS1 in cells. CHO-K1 cells were treated with PT $(3 \mu \mathrm{g} / \mathrm{ml})$ for $3 \mathrm{~h}$ (left) or $24 \mathrm{~h}$ (right) at $37^{\circ} \mathrm{C}$. Then, cells were fixed, and fluorescence-based PLA assay was performed according to the manufacturer's manual. PLA signals were counted from fluorescence pictures $(n=20$ images per condition from at least 2 independent experiments, mean \pm SD) with ImageJ. Values were normalized to the mean of the control samples. Significance was tested by Mann-Whitney test and refers to the respective untreated controls $\left({ }^{* * *} \mathrm{p}<0.0001\right)$. 
a
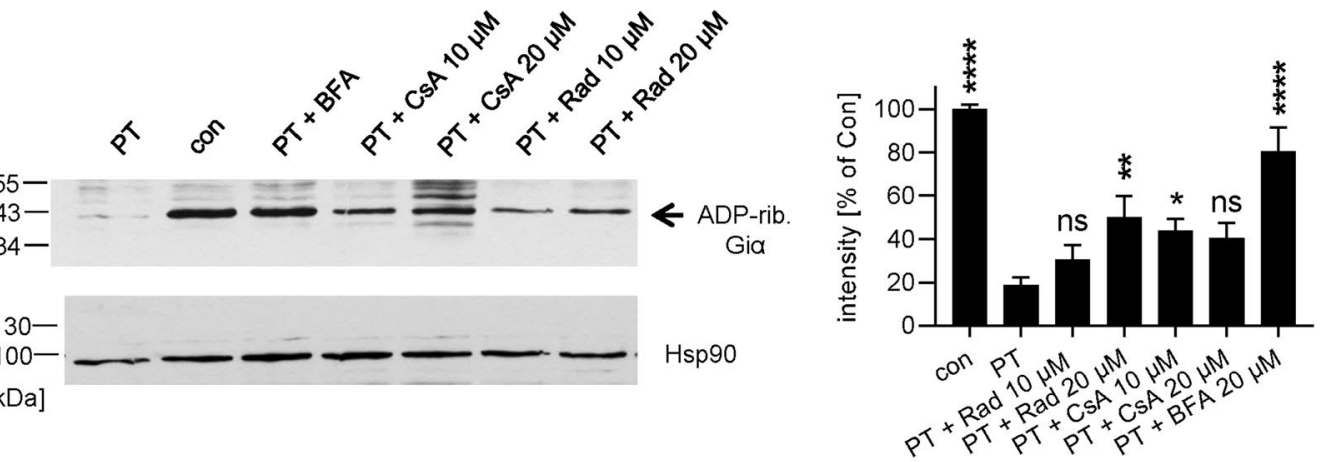

b
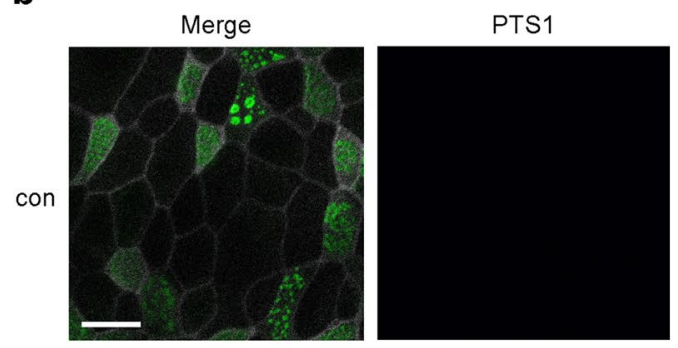

B-IV-tubulin
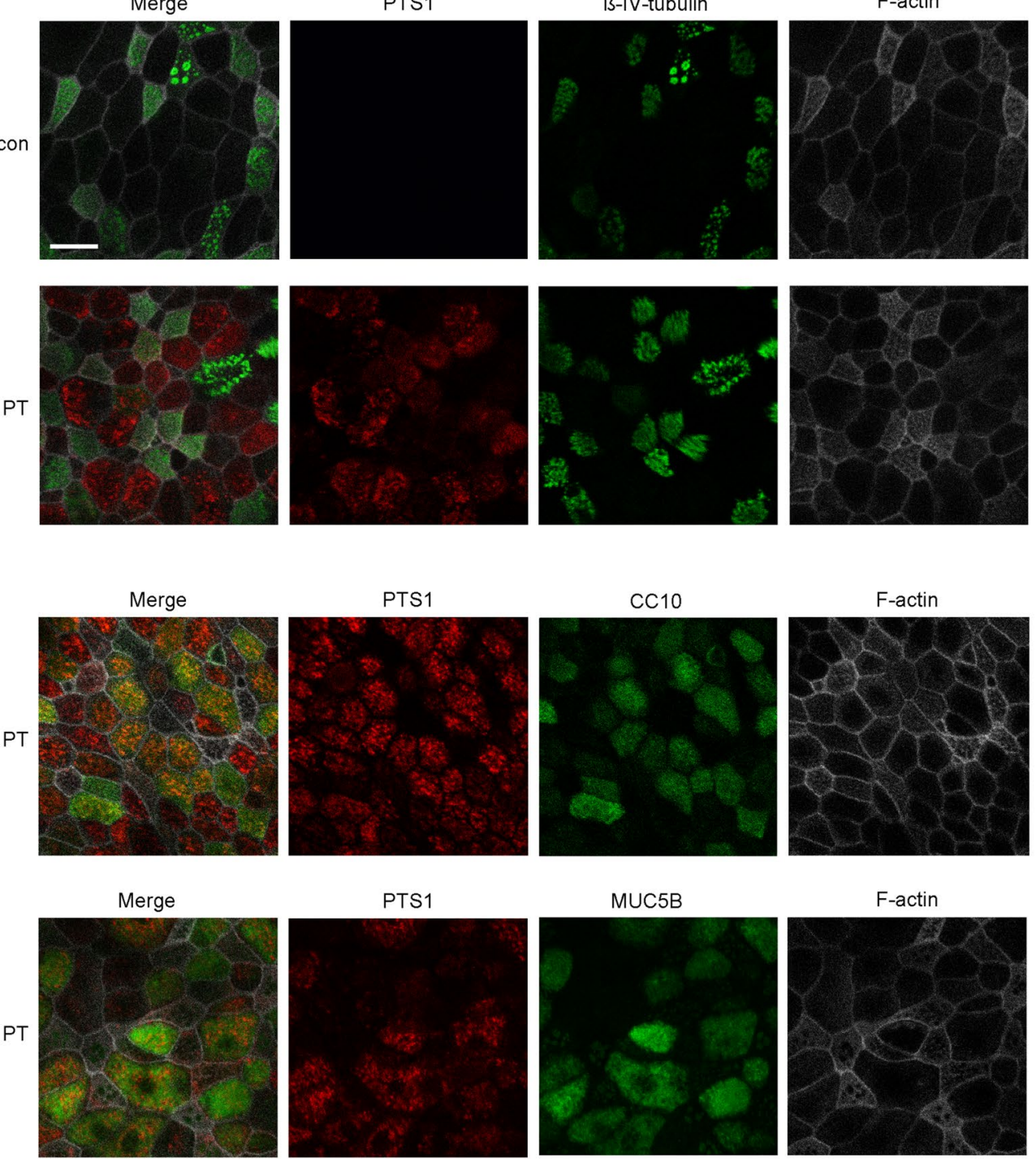

Figure 5. (a) Rad and CsA inhibit ADP-ribosylation of Gia in PT-treated basal cells. Basal cells were pre-incubated with Rad, CsA or BFA $(20 \mu \mathrm{M})$ for $45 \mathrm{~min}$ or left untreated for control (con). Subsequently, $10 \mathrm{ng} / \mathrm{ml} \mathrm{PT}$ was added for $4 \mathrm{~h}$ and the ADPribosylation status of Gia was determined as described before. A representative Western blot result is shown. Comparable protein loading was confirmed by Ponceau S (not shown) and Hsp90 staining. Western blot signals were quantified and normalized to protein loading $(n=8$ independent experiments, mean \pm SEM). Significance was tested by one-way ANOVA with Dunnett's multiple comparisons test and refers to samples treated with PT only $\left({ }^{* * *} \mathrm{p}<0.0001,{ }^{* *} \mathrm{p}<0.01,{ }^{*} \mathrm{p}<0.05, n s\right.$ not significant). For uncropped blots see Supplemental Fig. S13. (b) PTS1 is detected in secretory (CC10+, MUC5B+) cells but not in ciliated ( $\beta$-IVtubulin+) cells. hBAECs were incubated with PT $(20 \mu \mathrm{g} / \mathrm{ml})$ from the apical side for at least $72 \mathrm{~h}$ or left untreated for control. Cells were fixed with $4 \%$ PFA. For permeabilization and quenching of autofluorescence, cells were treated with $0.2 \%$ saponin. F-actin was stained with phalloidin-FITC. PTS1, $\beta$-IV-tubulin, CC10 and Muc5B were stained with specific primary and fluorescencelabeled secondary antibodies, respectively. Pictures were taken with an inverted confocal microscope. Scale bar $=20 \mu \mathrm{m}$. 


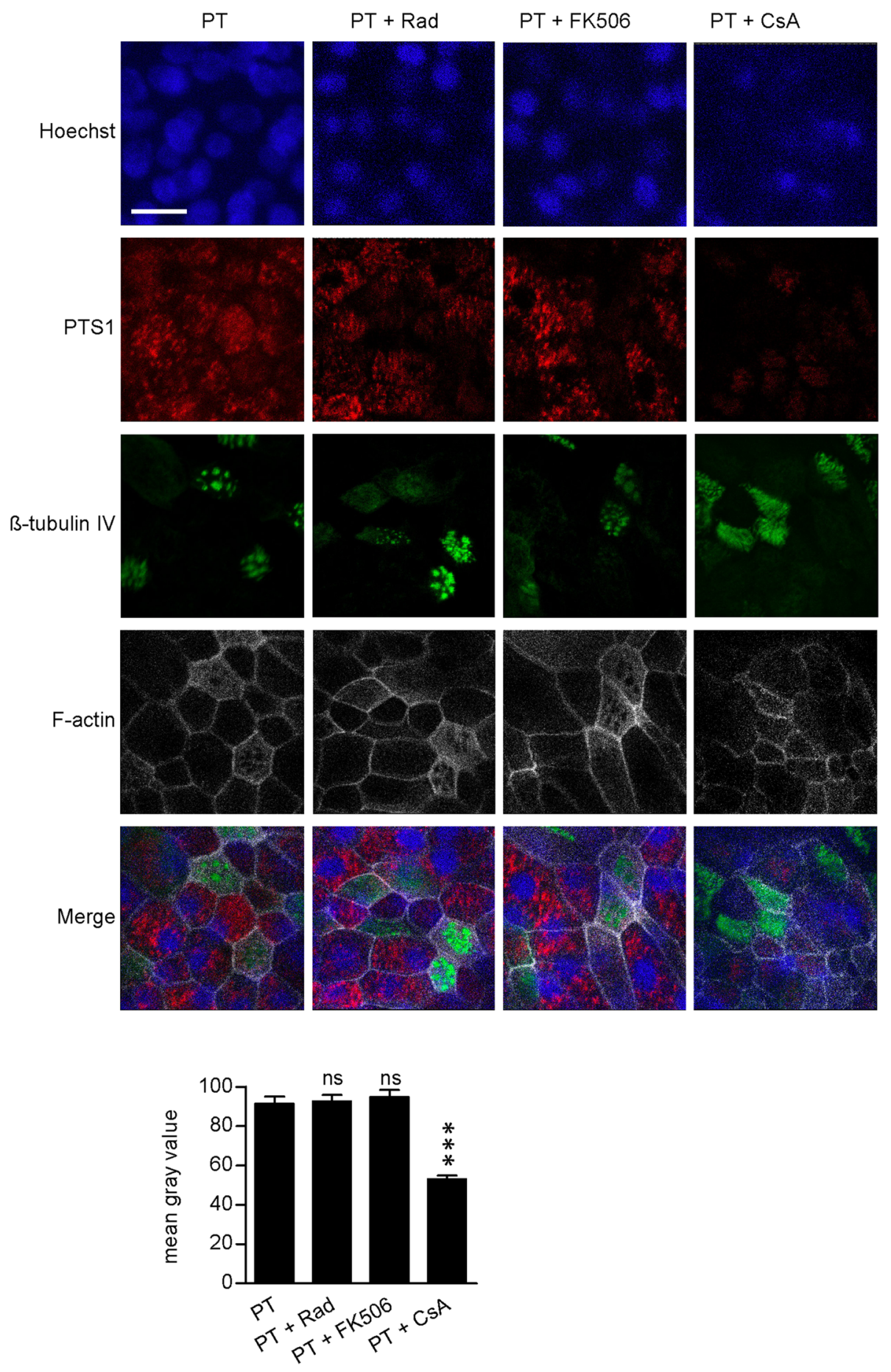

Figure 6. Effect of Rad, FK506 and CsA on uptake of PTS1 into hBAECs. hBAECs were pre-treated with respective inhibitors $(20 \mu \mathrm{M})$ and then incubated with PT $(20 \mu \mathrm{g} / \mathrm{ml})$ from the apical side for $72 \mathrm{~h}$. Cells were fixed with $4 \%$ PFA. For permeabilization and quenching of autofluorescence, cells were treated with $0.2 \%$ saponin. F-actin was stained with phalloidin-FITC. PTS1 and $\beta$-IV-tubulin were stained with specific primary and respective fluorescence-labeled secondary antibodies. Pictures were taken with a Cell Observer inverse microscope (Zeiss, Germany). Mean Gray Value Intensity was measured and plotted for PTS1 (after background subtraction) in PT and PT + inhibitor treated cultures. Values are given as mean \pm SEM ( $\mathrm{n}=60$ cells/condition). Significance was tested by Kruskal-Wallis test and refers to samples treated with only PT $\left({ }^{* *} \mathrm{p}<0.001, n s\right.$ not significant). Scale bar $=20 \mu \mathrm{m}$. 

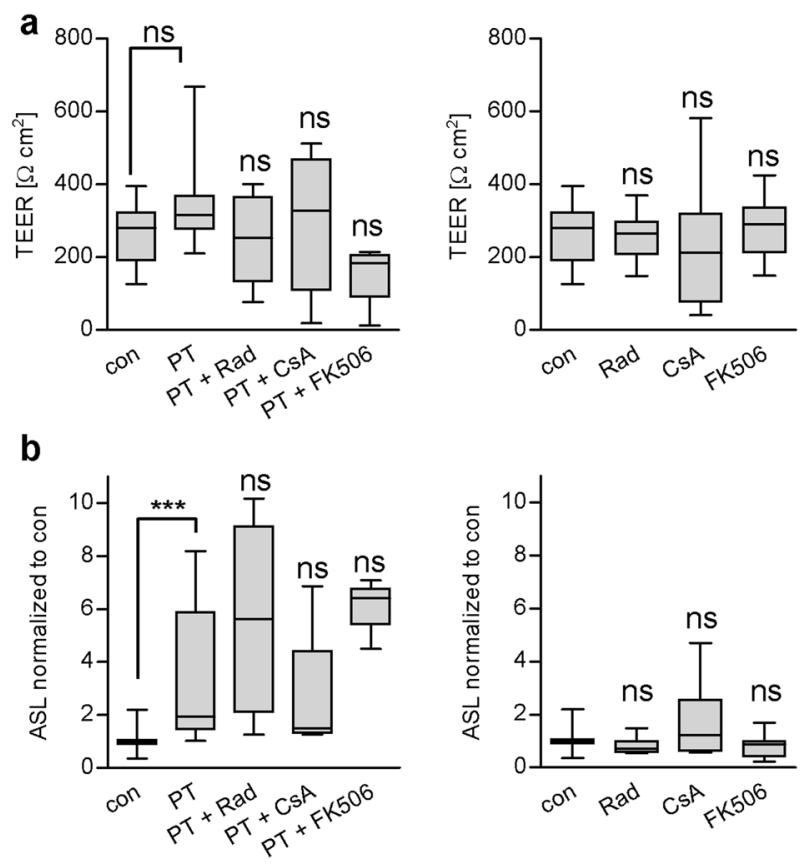

Figure 7. PT does not impair barrier function but increased apical surface liquid. (a) hBAECs were preincubated with Rad, CsA or FK506 $(20 \mu \mathrm{M})$ for 30 min or left untreated. Then $20 \mu \mathrm{g} / \mathrm{ml}$ PT was added for $72 \mathrm{~h}$ or cells were left untreated. Transepithelial electrical resistance (TEER) was measured using CellZScope from NanoAnalytics. (b) hBAECs were treated as described in A. After $72 \mathrm{~h}$ the apical surface liquid was determined. Values were obtained from at least two independent experiments $(\mathrm{n} \geq 4)$. Significance was tested by KruskalWallis test and refers to samples treated with only PT or as indicated ( ${ }^{* *} \mathrm{p}<0.001, n s$ not significant).

a

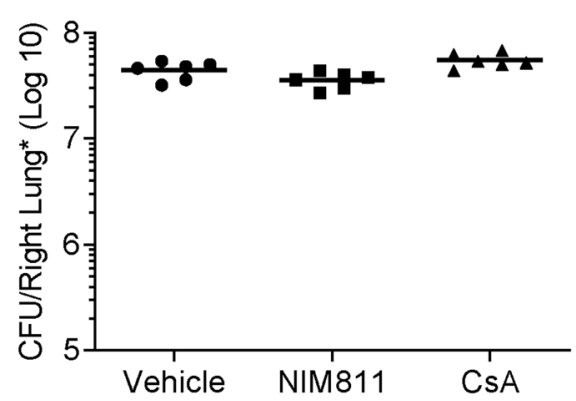

b

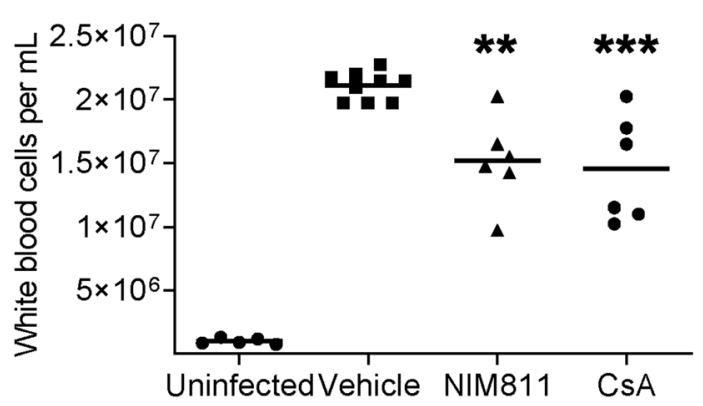

Figure 8. Cyclophilin inhibition reduces B. pertussis induced leukocytosis. Following aerosol infection 7-day old C57BL/6 pups were treated daily with vehicle, CsA $(25 \mathrm{mg} / \mathrm{kg})$ or NIM811 (25 mg/kg). No differences were determined in bacterial burden (a) however significant reduction in leukocytosis was noted in drug treated animals versus vehicle control (b). Each data point represents one animal. Significance was determined by oneway ANOVA with Dunnett's multiple comparisons test $\left({ }^{* *} \mathrm{p}<0.001,{ }^{* *} \mathrm{p}<0.01\right.$ vs. vehicle). Bars represent mean values.

Next, effects of PT on functional aspects of the human bronchial airway epithelium were investigated. PT showed no significant effect on trans-epithelial electrical resistance (TEER) of the human bronchial airway epithelium (Fig. 7a). However, PT significantly increased the apical surface liquid (ASL) of the functional human airway epithelium compared to untreated controls (Fig. 7b). This effect was not impaired by the chaperone inhibitors suggesting that other mechanisms might play a role that do not require PTS1 activity in the cytosol such as binding of the B-pentamer to the cell surface. Chaperone/PPIase inhibitors alone led to no significant effects on ASL or TEER values of treated samples compared to control (Fig. 7, right panels).

Cyp inhibitors reduce leukocytosis in an infant model of pertussis disease. Finally, effects of CsA and its non-immunosuppressive derivative NIM81 $1^{42}$ were investigated in an infant mouse model of pertussis. This model has recently been established and recapitulates several hallmarks of severe disease observed in humans such as leukocytosis and death, which are both PT-dependent ${ }^{8}$. Here, 7 -day old mice were infected 
with a PT-producing wild type strain of B. pertussis via aerosol and then treated intranasally with either CsA or NIM811 or vehicle. Treatment with CsA or NIM811 had no effect on the colony forming units detected from lung homogenates (Fig. 8). However, PT-induced leukocytosis was significantly reduced by CsA or NIM811 treatment in infant mice implicating a protective effect upon Cyp inhibition for the first time in vivo.

\section{Discussion}

Pertussis disease is a reemerging public health crisis for which there is currently no effective treatment. The current standard of care, treatment with macrolide antibiotics is only associated with improved symptoms when administered during the catarrhal stage of disease ${ }^{43}$. This is challenging as it is before the onset of the characteristic "whooping" cough. B. pertussis mediates disease through its ADP-ribosylating toxin (ADP-RT) PT. The ability of ADP-RTs to translocate their enzyme subunits to target cell cytosols has been the subject of much research (for review see ${ }^{44,45}$ ). We have shown other membrane translocating ADP-RTs, such as Corynebacterium diphtheriae diphtheria toxin, C. botulinum $\mathrm{C} 2$ toxin, $C$. perfringens iota toxin, and C. difficile CDT, require the chaperone activity of heat shock proteins Hsp90 and Hsp70, Cyps as well as PPIases from the FKBP family to transfer their enzymatic subunits from vesicular compartments to the cytosol of target cells ${ }^{37,40,46-53}$. Identifying commonalities in these toxins mechanisms of action may allow for the development of treatments which function for across diverse bacterial species.

Our previous work identified Cyp inhibitors as potent inhibitors of PT intoxication of mammalian cells ${ }^{29}$. Previously, we determined that Cyp isoforms CypA and Cyp40 interact with PTS1 in vitro and are required for uptake of PTS1 into the cytosol. This study revealed host Hsp90, Hsp70, Cyps and FKBPs are required for uptake of PTS1 into the cytosol. A direct and specific interaction of PTS1 with Hsp90, Hsp70, CypA, Cyp40, FKBP51 and FKBP52 was detected in vitro and interaction with FKBPs was partly mediated by the PPIase domain of these proteins. Human bronchial derived airway epithelial cells differentiated under air-liquid interface conditions allowed us to identify secretory cells as targets for PTS1 intoxication. Further, pharmacological inhibitors of Cyps protected against leukocytosis in an in vivo murine model of infant pertussis disease demonstrating the clinical potential of these molecules.

ADP-ribosylation of GTP-binding regulatory protein Gia by $\mathrm{PT}^{12}$ induces distinct morphological changes in CHO-K1 cells in a sensitive and reproducible manner ${ }^{35}$. This phenomenon is the basis of routine assays to determine monoclonal antibody function and to measure antitoxin responses ${ }^{54,55}$. Here, we utilized this CHO$\mathrm{K} 1$ assay and pharmacological inhibitors of heat shock proteins, Cyps and FKBPs to show PT interactions with Hsp90, Hsp70, FKBP and Cyps are required for PT-intoxication of CHO-K1 cells. Further, we determined inhibition of these host molecules prevents ADP-ribosylation of Gia protein and intracellular cAMP accumulation of $\mathrm{CHO}-\mathrm{K} 1$ by preventing uptake of PT into the cytosol, not by inhibiting PT enzymatic activity. Immobilization of the studied host chaperones and folding helper proteins on a nitrocellulose membrane and incubation with PTS1 identified Hsp90, Hsp70 and Hsc70, CypA, Cyp40, FKBP51 and FKBP52 as binding partners of PTS1 in a specific and concentration-dependent manner. Interestingly, Hsp90 requirement for translocation of PTS1 from the ER into the cytosol was shown in a previous study. In this study, PTS1 was expressed directly in the ER of transfected $\mathrm{CHO}$ cells and it was demonstrated that Hsp90-inhibition in these cells prevented translocation of PTS1 from the ER to the cytosol ${ }^{56}$. The effect of CsA, FK506 and VER on translocation of PTS1 from the ER to the cytosol was not investigated in such an isolated manner and therefore we cannot fully exclude an inhibiting effect of CsA, FK506 or VER on endocytosis or retrograde transport to the ER. Nevertheless, assistance of Cyps, FKBPs and Hsp70 during translocation into the cytosol as demonstrated for Hsp90 is conceivable.

Cyp40, FKBP51 and FKBP52 contain three TPR domains through which they bind to Hsp90 $0^{32,57}$ forming a Hsp90-multichaperone complex ${ }^{30,31}$. The Hsp90-multichaperone complex facilitates the stabilization, folding and activation of more than 200 mammalian proteins including steroid hormone receptors ${ }^{30,58-60}$. For folding and activation of steroid hormone receptors, activity of chaperones is required in a concerted and sequenced manner ${ }^{30}$. First, Hsp70 binds the unfolded protein and transfers it to an Hsp90 dimer. Subsequent binding of FKBPs or Cyps through TPR domains influences Hsp90 activity, in particular its ability to bind and hydrolyze ATP. ATP hydrolysis leads to conformational changes in Hsp90, which are transferred onto the unfolded protein resulting in a native folded protein. Since the same composition of cellular chaperones is required for the cytosolic delivery of ADP-RTs including PT it is conceivable that also in this case the chaperones act in a concerted manner.

Recently, it has been shown that cholera toxin requires Hsc/Hsp70 but not Cyps in addition to Hsp90 for its cellular uptake ${ }^{61}$. However, interaction of Hsc/Hsp70 and Hsp90 with CTA1, the enzyme domain of cholera toxin, occurs independently since both chaperones can bind simultaneously to CTA1, and binding occurs in the absence of the adapter protein Hop. This suggests an alternative interaction mechanism between cholera toxin and Hsp90/Hsc/Hsp70 that differs from the described Hsp90-multichaperone machinery.

We showed that interaction between Cyp40 and PTS1 was detected after $48 \mathrm{~h}$ after PT incubation. This suggests that Cyp40 not only facilitates the uptake of PTS1 into the cytosol but is required to stabilize PTS1 in an active conformation. A comparable mechanism is described for the cholera toxin, which also harbors ADPribosyltransferase activity and belongs to the group of long trip toxins. Hsp90 facilitates translocation of CTA1 to the cytosol, refolds CTA1 into an active conformation in an ATP-dependent manner and continues to bind to refolded CTA $1^{62,63}$. The finding that CTA 1 and PTS1 require the assistance of chaperones even after translocation and refolding might be due to the fact that both enzyme subunits are thermally unstable. This means that unfolding of CTA 1 and PTS1 occurs at $37^{\circ} \mathrm{C}$ after dissociation from the B-subunit. Therefore, releasing of toxin subunits from chaperones after translocation/refolding could again result in unfolding. Recently, two Hsp90 binding motifs in CTA1, which both are necessary for efficient translocation of CTA1 into the cytosol were identified ${ }^{56}$. One of these binding motifs was also identified in other ER-translocating ADP-ribosylating toxins including PT and Hsp90 requirement for PT uptake into the cytosol was demonstrated confirming and 
supporting the findings in the present study. Interestingly, the identified Hsp90-binding motif was absent in ADP-ribosylating toxins that translocate from endosomes suggesting an interaction mechanism distinct to some extent between short trip and long trip ADP-ribosylating toxins.

B. pertussis colonizes its host through attachment to the ciliated cells of the airways. It is known that PT can bind to various sialic acid-containing glycoproteins and various receptors have been found ${ }^{11,13}$. However, so far it is not clear which cell types are the target for PT in humans. Human bronchial airway epithelial basal cells differentiated in air-liquid interface conditions recapitulate the characteristics of the airway epithelial barrier. These epithelial cells differentiate into pseudostratified, polarized cells including ciliated and goblet (secretory) cells. From this, we determined that CC10 or MUC5B positive secretory cells, and not $\beta$-IV-tubulin positive ciliated cells were the target of PTS1 intoxication. It was determined that pharmacological inhibition of Cyps, but not Hsp90 or FKBP reduced detectable cytosolic PTS1 in these cells. Moreover, PT had no effect on the trans-epithelial resistance but increases the ASL of the differentiated human airway epithelium. These data suggest that PT does not impact gross epithelial barrier integrity but rather affects vectorial ion or fluid transport. The ASL shields the mucosal surface of lung epithelia and forms the first line of defense against airborne noxae and pathogens. A major task of lung epithelial cells is therefore to tightly regulate the ASL volume to maintain appropriate lung function and to ensure mucociliary clearance. ASL volume homeostasis is regulated by balancing water secretion and resorption. Secretion is driven by apical chloride channels (e.g. CFTR), whereas the epithelial $\mathrm{Na}^{+}$channel $(\mathrm{ENaC})$ mediates resorption. Hence, our observation that PT disturbs ASL homeostasis might reflect an impact on vectorial ion transport, either activating apical chloride channels or inhibiting ENaC. It has been demonstrated that PT intoxication results in increased intracellular cAMP levels, and CFTR is activated by intracellular cAMP ${ }^{64}$. Yet, future studies, including Ussing chamber measurements, will be required to decipher the impact of PT intoxication on transepithelial ion/water transport in more detail.

Results from another previous study in mice showed an upregulation of the epithelial anion exchanger pendrin in the lung after infection with $B$. pertussis ${ }^{65}$. Pendrin knockout mice showed reduced lung inflammatory pathology but even higher bacterial loads during infection suggesting a role of pendrin in the pathology. Pendrin exports bicarbonate to the ASL and thereby increases $\mathrm{pH}$, which possibly contributes to promoting the inflammatory pathology. These results indicate that manipulation of ASL parameters like volume or $\mathrm{pH}$ might be mediated by PT and play a role for the pathology and course of disease.

The mouse model of pertussis disease recapitulates the age-dependent nature of severe pertussis illness. Infant mice succumb to lethal infection at doses tolerated by adult mice. Leukocytosis, the rapid increase in vascular leukocytes, correlates with disease severity and lethality and is PT-mediated ${ }^{10,66}$. Infant mice, like humans, show robust increases in circulating leukocytes following infection. Here, we showed that inhibition of Cyp activity by CsA, a licensed drug with well-known pharmacokinetics and safety profiles ${ }^{67,68}$, and its non-immunosuppressive derivative NIM $811^{42}$ led to significantly decreased leukocytosis upon infection with PT-producing B. pertussis strains. To our knowledge, this is the first time that Cyps were pharmacologically targeted to successfully prevent cytotoxic effects of a bacterial AB-type toxin in an in vivo model. Together with our detailed mechanistic studies in $\mathrm{CHO}-\mathrm{K} 1$ cells and in vitro differentiated human airway epithelial model, these results provide a promising basis for the development of novel therapeutic strategies to prevent severe symptoms like leukocytosis caused by PT during $B$. pertussis infection.

\section{Methods}

Protein expression and purification. Recombinant proteins were expressed and purified as described before: Hsp70, Hsc70 ${ }^{37}$, Hsp90 ${ }^{51}$, FKBP12, FKBP51, FKBP52 ${ }^{52}$, CypA, Cyp40 ${ }^{40}$, FK1 domains of FKBP51 and FKBP52 $2^{53}$, C3bot ${ }^{69}$, PTS1 and $\mathrm{Gia}^{70}$.

Protein-protein interaction studies via dot blot system. Purified recombinant chaperones, PPIases and PPIase fragments were vacuum aspirated onto a nitrocellulose membrane as serial dilution starting with $1 \mu \mathrm{g} / \mathrm{ml}$ using the dot blot system (Bio-Rad, Feldkirchen, Germany). Recombinant C3 toxin from C. botulinum (C3bot) was chosen as a random protein to exclude unspecific protein-protein interactions. Successful transfer was confirmed by Ponceau S staining. Membrane was blocked with 5\% skim milk powder in PBS-Tween (PBST) and then cut and probed with PTS1 (Aviva Systems, San Diego, California, USA) or for control with PBST. After extensive washing, both membranes were incubated with anti-PTS1 antibody (Santa Cruz, Heidelberg, Germany) to detect bound PTS1 using HRP-coupled secondary antibody (Santa Cruz) in combination with ECL (enhanced chemiluminescence, Millipore, Merck, Darmstadt, Germany) system. Signals for both membranes were detected on the same X-ray film. Pictures were cropped for display purposes only.

Cell culture and intoxication experiments. Cell culture materials were obtained from Gibco unless indicated otherwise. Chinese hamster ovary cells strain $\mathrm{K} 1$ (CHO-K1, from DSMZ, Braunschweig, Germany) were cultivated in DMEM and HAM's F12 containing 5\% heat-inactivated fetal calf serum (Invitrogen, Thermo Fisher Scientific, Waltham, Massachusetts, USA), $1 \mathrm{mM}$ sodium-pyruvate and Penicillin-Streptomycin (PenStrep) (1:100) (Thermo Fisher Scientific). Cells were grown at $37^{\circ} \mathrm{C}$ and $5 \% \mathrm{CO}_{2}$ as described before ${ }^{29}$. Cells were trypsinized and reseeded every two to three days for at most 15-20 times. For intoxication experiments ${ }^{29,46,48}$, cells were seeded in culture dishes and the specific pharmacological inhibitors radicicol (inhibitor of ATPbinding site of Hsp90, Sigma-Aldrich, Merck, Darmstadt, Germany), cyclosporine A (inhibitor of Cyp activity, Sigma-Aldrich, Merck), FK506 (inhibitor of FKBP activity, Sigma-Aldrich, Merck), VER (inhibitor of ATPbinding site of Hsp70, Hsc70 and Grp78, Tocris Bioscience, Wiesbaden-Nordenstadt, Germany), HA9 (inhibitor of the substrate binding domain of Hsp70) ${ }^{37}$ and brefeldin A (disrupts Golgi apparatus, Sigma-Aldrich, Merck) were added for $30 \mathrm{~min}$. Then, PT (Sigma-Aldrich, Merck) was added either for $1 \mathrm{~h}$ with subsequent removal and 
further incubation for $18 \mathrm{~h}$ at $37^{\circ} \mathrm{C}$ or PT was added for $18 \mathrm{~h}$. Morphology of cells was documented by using a Zeiss Axiovert 40CFL microscope with a Jenoptik ProgRes C10 CCD camera. At least 3 images per treatment were taken and analyzed and the experiment was repeated independently at least 3 times. To quantify toxininduced effects on $\mathrm{CHO}-\mathrm{K} 1$ cells total number of cells per picture was manually counted using ImageJ (National Institutes of Health, Bethesda). Untreated control cells were set as $100 \%$ and values of other samples were calculated accordingly. Materials for cell culture experiments were obtained from TPP Techno Plastic Products (Trasadingen, Schweiz).

Primary human bronchial airway epithelial cell culture. Frozen primary human bronchial airway epithelial cells (hBAECs, Epithelix, Geneva, Switzerland) were obtained at passage 1. Of all cell types that are obtained from a human airway epithelium, only the basal cells survive. Basal cells were proliferated in growth medium (Promocell, Heidelberg, Germany) supplemented with Penicillin-Streptomycin (PenStrep, Thermo Fisher Scientific) until reaching $80 \%$ confluence in a T75 flask. Growth medium was replaced every two days. Cells were detached using DetachKIT (Promocell). $3.5 \times 10^{4}$ cells were seeded onto a transwell filter (Corning Costar 3470; Corning, Wiesbaden, Germany) pre-coated with collagen solution (StemCell Technologies, Cologne, Germany). Cells were maintained with $200 \mu$ growth medium in the apical and $600 \mu$ of growth medium in the basolateral compartment. To obtain a fully differentiated epithelium containing secretory and ciliated cells, apical medium was removed after $72 \mathrm{~h}$ and basolateral medium was switched to differentiation medium. Differentiation medium was a 50:50 mixture of DMEM-H without glutamine and pyruvate and LHC basal (both from Thermo Fisher Scientific) supplemented with supplement pack (Promocell), PenStrep and different trace elements as described in detail before ${ }^{71}$. Cells were grown until day 25 day with the basolateral medium being changed every three days. From day 14 onwards, cells were washed on the apical side every three days for 30 min with Dulbecco's phosphate buffered solution (DPBS, Biochrom, Berlin, Germany) to remove accumulated mucus. hBAECs cultures from 2-3 different donors were used for each experiment.

Sequential ADP-ribosylation of Gia in lysates from toxin-treated cells. CHO-K1 cells or human basal cells were pre-incubated with respective inhibitors and then intoxicated with PT for given incubation periods. Cells were lysed by freezing in ADP-ribosylation buffer (0.1 mM Tris- $\mathrm{HCl}$ (pH 7.6), $20 \mathrm{mM}$ DTT and $0.1 \mu \mathrm{M}$ ATP) or plus protease inhibitor complete (Roche, Basel, Switzerland) as described earlier ${ }^{17,29,70}$, followed by incubation with PTS1 (170 ng (of the commercially obtained PTS1) or $50 \mathrm{nM}$ (of the recombinantly expressed $\mathrm{rPTxS} 1))$ and biotin-labeled $\mathrm{NAD}^{+}(10 \mu \mathrm{M}$ or $1 \mu \mathrm{M}$, Trevigen, Gaithersburg, Maryland, USA) for 40-60 min at room temperature for in vitro ADP-ribosylation of Gia, which had not yet been ADP-ribosylated by PT during the previous incubation. Samples were subjected to SDS-PAGE, blotted and ADP-ribosylated, i.e. biotin-labeled, Gia detected with streptavidin-peroxidase (Strep-POD, Sigma-Aldrich, Merck) using the ECL system. Comparable amounts of protein were confirmed by Ponceau-S-staining. Densitometric quantification of Western blot signals was measured using Adobe Photoshop CS6 and values were normalized on the amount of loaded protein.

iGIST bioassay. The iGIST bioassay was performed as previously described ${ }^{38}$. In brief, HEK293 cells that have been stably transfected with Gia-coupled somatostatin receptor 2 (SSTR2) GPCR were seeded as 60,000 cells/well into 96-well plates with light-tight walls in DMEM/F-12 (1:1) medium (Gibco), supplemented with fetal calf serum (10\%) and Pen-Strep (1\%) 1 day prior to the experiment. Cells were pre-incubated with inhibitors $(5 \mu \mathrm{M})$ or matched solvents (DMSO or ethanol) for $30 \mathrm{~min}$ and then challenged with PT $(500 \mathrm{ng} / \mathrm{ml}) \mathrm{or}$ matched buffer SolC (both in 50\% glycerol, $50 \mathrm{mM}$ TRIS, $10 \mathrm{mM}$ glycine, $0.5 \mathrm{M} \mathrm{NaCl}$, pH 7.5) for $3 \mathrm{~h}$. After addition of the inducing medium containing the luminescent GloSensor cAMP reagent (Promega, Walldorf, Germany), baseline luminescence was recorded for $15 \mathrm{~min}$ at the Orion Microplate Luminometer (Berthold Detection Systems). To activate the adenylate cyclase, forskolin (Sigma-Aldrich, Merck) was added and at the same time octreotide (Bachem, Bubendorf, Switzerland) was applied to stimulate the Gia-coupled SSTR2 GPCR. After spiking, luminescence was measured for $1 \mathrm{~h}$.

In vitro enzyme activity of PTS1. Recombinant Gia $(0.5 \mu \mathrm{M})$ was incubated for 30 min with $10 \mu \mathrm{M}$ Rad, CsA, $20 \mu \mathrm{M}$ FK506 or VER at room temperature. As a control Gia was incubated with buffer (50 nM sodium phosphate buffer, $\mathrm{pH} 7)$ only. After $30 \mathrm{~min} \operatorname{rPTxS1}^{70}(50 \mathrm{nM})$ was added together with biotin-labeled NAD ${ }^{+}$ $(1 \mu \mathrm{M}$, Trevigen) and incubated for $40 \mathrm{~min}$ at room temperature. Samples were subjected to SDS-PAGE and blotted onto a nitrocellulose membrane. ADP-ribosylated, i.e. biotin-labeled, Gia was detected with Strep-POD. Comparable amounts of protein were confirmed by Ponceau-S-staining.

Toxin binding assay. CHO-K1 cells were pre-incubated with $10 \mu \mathrm{M}$ Rad, CsA, FK506 or $30 \mu \mathrm{M}$ VER. The assay was performed as previously described ${ }^{29,46}$. Cells were cooled down to $4^{\circ} \mathrm{C}$ for 15 min to prevent endocytosis. $500 \mathrm{ng} / \mathrm{ml} \mathrm{PT}$ were added, and cells were incubated for $30 \mathrm{~min}$ at $4{ }^{\circ} \mathrm{C}$. Subsequently, culture medium was removed, and cells were washed with PBS to remove unbound PT. Then cells were scraped with boiling SDSsample buffer and heated for $10 \mathrm{~min}$ at $95^{\circ} \mathrm{C}$. SDS-PAGE and Western Blotting was performed. To detect cellbound PT an antibody against the S1 subunit of PT and a peroxidase-coupled secondary antibody were used.

Immunofluorescence. Fluorescence microscopy was performed as described earlier ${ }^{29,46}$. Cells were fixed after intoxication experiments with $4 \%$ paraformaldehyde (PFA), permeabilized with Triton-X $100(0.4 \%$ in PBS), treated with glycine (100 nM in PBS) and blocked with 5\% skim milk powder or $10 \%$ normal goat serum 
(Jackson ImmunoResearch, West Grove, Pennsylvania, USA) and 1\% BSA in PBST for $1 \mathrm{~h}$ at $37^{\circ} \mathrm{C}$. Samples were incubated with anti-PTS1 antibody (Santa Cruz, 1:50 diluted in 10\% NGS and 1\% BSA in PBST) for $1 \mathrm{~h}$ at $37^{\circ} \mathrm{C}$. After washing, fluorescence-labeled secondary antibody anti-mouse 568 (goat) (Invitrogen) was added ( $1: 750$ diluted in $10 \%$ NGS and $1 \%$ BSA in PBST) for $1 \mathrm{~h}$ at $37^{\circ} \mathrm{C}$. Nuclei were stained with Hoechst and F-actin with phalloidin-FITC (Sigma-Aldrich, Merck). Between the individual working steps, cells were washed with PBS. Fluorescence imaging was performed with the iMIC Digital Microscope (FEI Munich, Germany) using the Live Acquisition 2.6 software (FEI Munich) and processed with ImageJ 1.4.3.47 software (National Institutes of Health, Bethesda).

hBAECs grown on transwell filters were fixed for $15 \mathrm{~min}$ in $4 \%$ paraformaldehyde in DPBS. Cells were then permeabilized and blocked for $5 \mathrm{~min}$ in DPBS containing $0.2 \%$ saponin and 10\% FBS (Thermo Fisher Scientific). Cells were stained with primary (1:25-50) and secondary (1:400) antibodies in DPBS, $0.2 \%$ saponin and $10 \%$ FBS. The $\alpha$ - $\beta$-IV rabbit monoclonal antibody (ab179509) and ZO-1 (ab99462) were purchased from Abcam (Cambridge, United Kingdom), $\alpha$-club cell secretory protein rat monoclonal antibody (MAB4218) was from R\&D Systems (Minneapolis, Minnesota, USA) and the $\alpha$-Muc5B rabbit polyclonal antibody (HPA008246) was from Sigma-Aldrich. Fluorescently labeled secondary antibodies were obtained from Molecular Probes (Thermo Fisher Scientific). Alexa Fluor 647-Phalloidin (8940) was obtained from NEB. Images were taken on an inverted confocal microscope (Leica TCS SP5, Leica, Wetzlar, Germany) using a $63 \times$ lens (Leica HCX PL APO lambda blue $63.0 \times 1.40$ OIL UV). Images for the blue (DAPI), green (AlexaFluor 488), red (AlexaFluor 568) and far-red (AlexaFluor 647) channels were taken in sequential mode using appropriate excitation and emission settings.

Protein interaction analysis in cultured cells by Duolink using proximity ligation assay (PLA). CHO-K1 cells were incubated with PT for indicated periods of time. Cells were fixed with $4 \%$ PFA, permeabilized and blocked with $10 \%$ NGS and 1\% BSA in PBST. Subsequently, cells were incubated with mouse anti-PTS1 (Santa Cruz or Abcam) and rabbit anti-Cyp40 (Thermo Fisher Scientific), rabbit anti-Hsp90 (Thermo Fisher Scientific), rabbit anti-Hsp70 (Enzo Life Sciences) or rabbit anti-FKBP51 (Santa Cruz) antibodies for $1 \mathrm{~h}$ at $37^{\circ} \mathrm{C}$. PLA assay was performed according to the manufacturer's protocol (Duolink using PLA technology, Sigma-Aldrich, Merck) and as described before ${ }^{46,47}$.

Measurement of the transepithelial electrical resistance (TEER). TEER was analyzed by impedance spectrometry using the cellZscope (NanoAnalytics, Münster, Germany). For measurements, the basal electrode was overlaid by $500 \mu \mathrm{l}$ equilibrated DMEM-H medium filters inserted and $250 \mu \mathrm{l}$ ediuDMEM-H medium was added to the apical side of the filter. Measurements were performed immediately after positioning of apical electrodes. Data were acquired and analyzed using the software provided with the instrument (NanoAnalytics).

Measurement of apical surface liquid (ASL) volume. ASL measurements were performed using the $\mathrm{D}_{2} \mathrm{O}$ dilution method as described previously ${ }^{72}$. Filters with confluent cell layers were placed in $500 \mu \mathrm{l}$ of differentiation medium containing respective inhibitors and pre-incubated for $30 \mathrm{~min}$ at $37^{\circ} \mathrm{C} .25 \mu \mathrm{l}$ of isotonic $\mathrm{NaCl}$, containing respective inhibitors, was added to the apical compartment. Silicon sealed control filters loaded with $25 \mu \mathrm{l}$ isotonic $\mathrm{NaCl}$ solution were randomly distributed throughout the plate to estimate volume changes caused by evaporation. After $72 \mathrm{~h}$ apical fluid volumes were collected with $25 \mu \mathrm{D} \mathrm{D}_{2} \mathrm{O}$ containing $0.9 \%$ (w/v) $\mathrm{NaCl}$ for analysis. Water concentrations were measured by attenuated total reflexion mid-infrared spectroscopy on A Vertex 70 FT-IR spectrometer, equipped with a BioATRCell-II unit and a liquid nitrogen cooled MCT detector (Bruker Optics, Fällanden, Switzerland), to calculate ASL volumes. Data acquisition and processing was performed using OPUS 6.5 (Bruker Optics). Before each measurement a background spectrum of the empty ATR-unit was collected and a calibration series $(0 \%, 15 \%, 25 \%, 40 \%, 50 \%, 65 \%$ (v/v) of isotone $\mathrm{NaCl}$ solution in $\mathrm{H}_{2} \mathrm{O}$ and $0.9 \% \mathrm{NaCl}$ solution in $\mathrm{D}_{2} \mathrm{O}$ ) was measured. Areas below absorption bands were blotted against water concentration and linear regression was calculated through data points to obtain the slope $(m)$ and $y$-interception $\left(y_{0}\right)$, to calculate water concentrations as described in detail before ${ }^{72}$. The apical volume $\left(V_{\text {api }}\right)$ was calculated according to:

$$
V_{a p i}=C_{\mathrm{H}_{2} \mathrm{O}} \times V_{\mathrm{D}_{2} \mathrm{O}} /\left(100 \%-C_{\mathrm{H}_{2} \mathrm{O}}\right)
$$

with $V_{D_{2} O}$ being the volume $(25 \mu \mathrm{l})$ of $\mathrm{D}_{2} \mathrm{O}$ in $0.9 \% \mathrm{NaCl}$. Changes in apical volume $\left(\Delta V_{a p i}\right)$ were calculated by subtracting the remaining volume from the initial volume ( $25 \mu \mathrm{l}$ isotonic $\mathrm{NaCl}$ solution). Changes in apical volumes in experimental filters were corrected for changes in evaporation controls.

Infant mouse studies. 7-day old C57BL/6 mice were used in accordance with the University of Maryland, Baltimore Institutional Animal Care and Use Committee. A streptomycin resistant derivative of the Tohama I "wild-type" B. pertussis strain was grown on Bordet-Gengou (BG) agar plates supplemented with defibrinated sheep blood (10\%, Lampire Biological Products, Pipersville, Pennsylvania, USA) and $200 \mu \mathrm{g} / \mathrm{ml}$ streptomycin (Sigma-Aldrich, Merck). All comparisons were made between littermates pre-designated as receiving vehicle or one of the inhibitors. When dividing mice into groups attempts were made to match bodyweights between groups instead of true randomization. Tails were marked with colored pens daily to distinguish experimental groups within a litter. Only litters between sized between 5 and 8 pups were used to minimize variability associated with litter size. Animals were challenged via aerosol administered by a nebulizer system (Pari Vios) for twenty minutes. Lungs were excised post-euthanasia, homogenized mechanically (Omni International, Tulsa, Oklahoma, USA) and bacterial burden determined by plating on BG agar. Animals were intranasally administered vehicle (Cremophor:Ethanol:Saline 10:1:89), CsA (25 mg/kg, Sigma-Aldrich, Merck) or NIM811 (25 mg/ 
kg, MedChemExpress, Monmouth, New Jersey, USA). To measure leukocytosis blood was harvested 7 days post-infection by cardiac puncture into ethylenediaminetetraacetic acid (EDTA) containing $1.7 \mathrm{ml}$ tubes (Fisher Scientific) and treated with ammonium-chloride-potassium (ACK) lysis buffer to lyse red blood cells. The investigator performing the harvest then assigned the samples numbers unrelated to their group. White blood cells were then counted using a hemocytometer by an investigator blind to sample identifications. Inhibitor treated mice were compared to vehicle treated animals by ANOVA with Dunnett's multiple comparisons test using GraphPad Prism. Studies were performed in both male and female mice, but exact numbers of each gender could not be determined at time of infection (7-days-old).

Ethics statement. Animals were used in accordance with the University of Maryland Institutional Animal Care and Use Committee protocol 0417005 (University of Maryland, Baltimore, MD) following approval by the Office of Animal Welfare Assurance. Animals were euthanized by $\mathrm{CO}_{2}$ asphyxiation followed by cervical dislocation and thoracotomy. All methods were performed in accordance with the relevant guidelines and regulations. The study was carried out in compliance with the ARRIVE guidelines.

Received: 3 August 2020; Accepted: 16 February 2021

Published online: 08 March 2021

\section{References}

1. Kilgore, P. E., Salim, A. M., Zervos, M. J. \& Schmitt, H.-J. Pertussis: Microbiology, disease, treatment, and prevention. Clin. Microbiol. Rev. 29, 449-486 (2016).

2. Mattoo, S. \& Cherry, J. D. Molecular pathogenesis, epidemiology, and clinical manifestations of respiratory infections due to Bordetella pertussis and other Bordetella subspecies. Clin. Microbiol. Rev. 18, 326-382 (2005).

3. Carbonetti, N. H. Pertussis leukocytosis: Mechanisms, clinical relevance and treatment. Pathog. Dis. 74, ftw087 (2016).

4. Heininger, U., Klich, K., Stehr, K. \& Cherry, J. D. Clinical findings in Bordetella pertussis infections: results of a prospective multicenter surveillance study. Pediatrics 100, E10 (1997).

5. Publication, W. H. O. Pertussis vaccines: WHO position paper-recommendations. Vaccine 29, 2355-2356 (2011).

6. Domenech de Cellès, M., Magpantay, F. M. G., King, A. A. \& Rohani, P. The pertussis enigma: Reconciling epidemiology, immunology and evolution. Proc. Biol. Sci. 283, 20152309 (2016).

7. Carbonetti, N. H. Contribution of pertussis toxin to the pathogenesis of pertussis disease. Pathog. Dis. 73, ftv73 (2015).

8. Scanlon, K. M., Snyder, Y. G., Skerry, C. \& Carbonetti, N. H. Fatal pertussis in the neonatal mouse model is associated with pertussis toxin-mediated pathology beyond the airways. Infect. Immun. 85, e00355-17 (2017).

9. Pittman, M. The concept of pertussis as a toxin-mediated disease. Pediatr. Infect. Dis. 3, 467-486 (1984).

10. Scanlon, K., Skerry, C. \& Carbonetti, N. Association of pertussis toxin with severe pertussis disease. Toxins 11, 373 (2019).

11. Stein, P. E. et al. The crystal structure of pertussis toxin. Struct. Lond. Engl. 1993(2), 45-57 (1994).

12. Tamura, M. et al. Subunit structure of islet-activating protein, pertussis toxin, in conformity with the A-B model. Biochemistry 21, 5516-5522 (1982).

13. Locht, C., Coutte, L. \& Mielcarek, N. The ins and outs of pertussis toxin. FEBS J. 278, 4668-4682 (2011).

14. Witvliet, M. H., Burns, D. L., Brennan, M. J., Poolman, J. T. \& Manclark, C. R. Binding of pertussis toxin to eucaryotic cells and glycoproteins. Infect. Immun. 57, 3324-3330 (1989).

15. Lippincott-Schwartz, J., Yuan, L. C., Bonifacino, J. S. \& Klausner, R. D. Rapid redistribution of Golgi proteins into the ER in cells treated with brefeldin A: Evidence for membrane cycling from Golgi to ER. Cell 56, 801-813 (1989).

16. Plaut, R. D. \& Carbonetti, N. H. Retrograde transport of pertussis toxin in the mammalian cell. Cell Microbiol. 10, 1130-1139 (2008).

17. Xu, Y. \& Barbieri, J. T. Pertussis toxin-mediated ADP-ribosylation of target proteins in Chinese hamster ovary cells involves a vesicle trafficking mechanism. Infect. Immun. 63, 825-832 (1995).

18. Hazes, B., Boodhoo, A., Cockle, S. A. \& Read, R. J. Crystal structure of the pertussis toxin-ATP complex: A molecular sensor. J. Mol. Biol. 258, 661-671 (1996).

19. Plaut, R. D., Scanlon, K. M., Taylor, M., Teter, K. \& Carbonetti, N. H. Intracellular disassembly and activity of pertussis toxin require interaction with ATP. Pathog. Dis. 74, ftw065 (2016).

20. Murrell-Lagnado, R. D. \& Frick, M. P2X4 and lysosome fusion. Curr. Opin. Pharmacol. 47, 126-132 (2019).

21. Banerjee, T. et al. Thermal unfolding of the pertussis toxin $\mathrm{S} 1$ subunit facilitates toxin translocation to the cytosol by the mechanism of endoplasmic reticulum-associated degradation. Infect. Immun. 84, 3388-3398 (2016).

22. Pande, A. H., Moe, D., Jamnadas, M., Tatulian, S. A. \& Teter, K. The pertussis toxin S1 subunit is a thermally unstable protein susceptible to degradation by the 20 S proteasome. Biochemistry $45,13734-13740$ (2006).

23. Worthington, Z. E. V. \& Carbonetti, N. H. Evading the proteasome: Absence of lysine residues contributes to pertussis toxin activity by evasion of proteasome degradation. Infect. Immun. 75, 2946-2953 (2007).

24. Bokoch, G. M., Katada, T., Northup, J. K., Hewlett, E. L. \& Gilman, A. G. Identification of the predominant substrate for ADPribosylation by islet activating protein. J. Biol. Chem. 258, 2072-2075 (1983).

25. Katada, T. \& Ui, M. Direct modification of the membrane adenylate cyclase system by islet-activating protein due to ADPribosylation of a membrane protein. Proc. Natl. Acad. Sci. U.S.A. 79, 3129-3133 (1982).

26. Kirimanjeswara, G. S., Agosto, L. M., Kennett, M. J., Bjornstad, O. N. \& Harvill, E. T. Pertussis toxin inhibits neutrophil recruitment to delay antibody-mediated clearance of Bordetella pertussis. J. Clin. Investig. 115, 3594-3601 (2005).

27. Spangrude, G. J., Sacchi, F., Hill, H. R., Van Epps, D. E. \& Daynes, R. A. Inhibition of lymphocyte and neutrophil chemotaxis by pertussis toxin. J. Immunol. Baltim. Md 1950(135), 4135-4143 (1985).

28. Andreasen, C. \& Carbonetti, N. H. Pertussis toxin inhibits early chemokine production to delay neutrophil recruitment in response to Bordetella pertussis respiratory tract infection in mice. Infect. Immun. 76, 5139-5148 (2008).

29. Ernst, K. et al. Pharmacological cyclophilin inhibitors prevent intoxication of mammalian cells with bordetella pertussis toxin. Toxins 10, 181 (2018).

30. Li, J. \& Buchner, J. Structure, function and regulation of the hsp90 machinery. Biomed. J. 36, 106-117 (2013).

31. Pratt, W. B. \& Toft, D. O. Regulation of signaling protein function and trafficking by the hsp90/hsp70-based chaperone machinery. Exp. Biol. Med. Maywood NJ 228, 111-133 (2003).

32. Schiene-Fischer, C. Multidomain peptidyl prolyl cis/trans isomerases. Biochim. Biophys. Acta https://doi.org/10.1016/j.bbage n.2014.11.012 (2014). 
33. Porter, G. A. \& Beutner, G. Cyclophilin D, somehow a master regulator of mitochondrial function. Biomolecules 8, 176 (2018).

34. Gestal, M. C., Johnson, H. M. \& Harvill, E. T. Immunomodulation as a novel strategy for prevention and treatment of Bordetella spp. infections. Front. Immunol. 10, 2869 (2019).

35. Hewlett, E. L., Sauer, K. T., Myers, G. A., Cowell, J. L. \& Guerrant, R. L. Induction of a novel morphological response in Chinese hamster ovary cells by pertussis toxin. Infect. Immun. 40, 1198-1203 (1983).

36. el Bayâ, A., Linnemann, R., von Olleschik-Elbheim, L., Robenek, H. \& Schmidt, M. A. Endocytosis and retrograde transport of pertussis toxin to the Golgi complex as a prerequisite for cellular intoxication. Eur. J. Cell Biol. 73, 40-48 (1997).

37. Ernst, K. et al. A novel Hsp70 inhibitor prevents cell intoxication with the actin ADP-ribosylating Clostridium perfringens iota toxin. Sci. Rep. 6, 20301 (2016).

38. Paramonov, V. M., Sahlgren, C., Rivero-Müller, A. \& Pulliainen, A. T. iGIST-A kinetic bioassay for pertussis toxin based on its effect on inhibitory GPCR signaling. ACS Sens. 5, 3438-3448 (2020).

39. Dmochewitz, L. et al. Role of CypA and Hsp90 in membrane translocation mediated by anthrax protective antigen. Cell Microbiol. 13, 359-373 (2011).

40. Ernst, K. et al. Cyclophilin-facilitated membrane translocation as pharmacological target to prevent intoxication of mammalian cells by binary clostridial actin ADP-ribosylated toxins. J. Mol. Biol. 427, 1224-1238 (2015).

41. Okuda, K. et al. Localization of secretory mucins MUC5AC and MUC5B in normal/healthy human airways. Am. J. Respir. Crit. Care Med. 199, 715-727 (2019).

42. Rosenwirth, B. et al. Inhibition of human immunodeficiency virus type 1 replication by SDZ NIM 811, a nonimmunosuppressive cyclosporine analog. Antimicrob. Agents Chemother. 38, 1763-1772 (1994).

43. Altunaiji, S., Kukuruzovic, R., Curtis, N. \& Massie, J. Antibiotics for whooping cough (pertussis). Cochrane Database Syst. Rev. https://doi.org/10.1002/14651858.CD004404.pub3 (2007).

44. Barth, H. \& Ernst, K. Chaperones and ADP-ribosylating bacterial toxins. In Microbial Toxins (eds Gopalakrishnakone, P. et al.) 1-22 (Springer, Dordrecht, 2016). https://doi.org/10.1007/978-94-007-6725-6_7-1.

45. Ernst, K., Schnell, L. \& Barth, H. Host cell chaperones Hsp70/Hsp90 and peptidyl-prolyl cis/trans isomerases are required for the membrane translocation of bacterial ADP-ribosylating toxins. Curr. Top. Microbiol. Immunol. 406, 163-198 (2017).

46. Ernst, K. et al. Hsp70 facilitates trans-membrane transport of bacterial ADP-ribosylating toxins into the cytosol of mammalian cells. Sci. Rep. 7, 2724 (2017).

47. Ernst, K., Kling, C., Landenberger, M. \& Barth, H. Combined pharmacological inhibition of cyclophilins, FK506-binding proteins, Hsp90, and Hsp70 protects cells from Clostridium botulinum C2 toxin. Front. Pharmacol. 9, 1287 (2018).

48. Haug, G. et al. The host cell chaperone Hsp90 is essential for translocation of the binary Clostridium botulinum $\mathrm{C} 2$ toxin into the cytosol. J. Biol. Chem. 278, 32266-32274 (2003).

49. Haug, G., Aktories, K. \& Barth, H. The host cell chaperone Hsp90 is necessary for cytotoxic action of the binary iota-like toxins. Infect. Immun. 72, 3066-3068 (2004).

50. Kaiser, E., Pust, S., Kroll, C. \& Barth, H. Cyclophilin A facilitates translocation of the Clostridium botulinum C2 toxin across membranes of acidified endosomes into the cytosol of mammalian cells. Cell Microbiol. 11, 780-795 (2009).

51. Kaiser, E. et al. Membrane translocation of binary actin-ADP-ribosylating toxins from Clostridium difficile and Clostridium perfringens is facilitated by cyclophilin A and Hsp90. Infect. Immun. 79, 3913-3921 (2011).

52. Kaiser, E. et al. FK506-binding protein 51 interacts with Clostridium botulinum C2 toxin and FK506 inhibits membrane translocation of the toxin in mammalian cells. Cell Microbiol. 14, 1193-1205 (2012).

53. Schuster, M. et al. The Hsp90 machinery facilitates the transport of diphtheria toxin into human cells. Sci. Rep. 7, 613 (2017).

54. Dalby, T., Sørensen, C., Petersen, J. W. \& Krogfelt, K. A. Pertussis serology: Assessment of IgG anti-PT ELISA for replacement of the CHO cell assay. APMIS Acta Pathol. Microbiol. Immunol. Scand. 118, $968-972$ (2010).

55. Markey, K., Asokanathan, C. \& Feavers, I. Assays for determining pertussis toxin activity in acellular pertussis vaccines. Toxins 11, 417 (2019).

56. Kellner, A., Taylor, M., Banerjee, T., Britt, C. B. T. \& Teter, K. A binding motif for Hsp90 in the A chains of ADP-ribosylating toxins that move from the endoplasmic reticulum to the cytosol. Cell Microbiol. https://doi.org/10.1111/cmi.13074 (2019).

57. Ratajczak, T. \& Carrello, A. Cyclophilin 40 (CyP-40), mapping of its hsp90 binding domain and evidence that FKBP52 competes with CyP-40 for hsp90 binding. J. Biol. Chem. 271, 2961-2965 (1996).

58. McClellan, A. J. et al. Diverse cellular functions of the Hsp90 molecular chaperone uncovered using systems approaches. Cell 131, 121-135 (2007).

59. Millson, S. H. et al. A two-hybrid screen of the yeast proteome for $\mathrm{Hsp} 90$ interactors uncovers a novel Hsp 90 chaperone requirement in the activity of a stress-activated mitogen-activated protein kinase, Slt2p (Mpk1p). Eukaryot. Cell 4, 849-860 (2005).

60. Zhao, R. et al. Navigating the chaperone network: an integrative map of physical and genetic interactions mediated by the hsp 90 chaperone. Cell 120, 715-727 (2005).

61. Burress, H., Kellner, A., Guyette, J., Tatulian, S. A. \& Teter, K. HSC70 and HSP90 chaperones perform complementary roles in translocation of the cholera toxin A1 subunit from the endoplasmic reticulum to the cytosol. J. Biol. Chem. https://doi.org/10.1074/ jbc.RA119.008568 (2019).

62. Burress, H., Taylor, M., Banerjee, T., Tatulian, S. A. \& Teter, K. Co- and post-translocation roles for HSP90 in cholera intoxication. J. Biol. Chem. 289, 33644-33654 (2014).

63. Taylor, M. et al. Hsp90 is required for transfer of the cholera toxin A1 subunit from the endoplasmic reticulum to the cytosol. J. Biol. Chem. 285, 31261-31267 (2010).

64. Csanády, L., Vergani, P. \& Gadsby, D. C. Structure, gating, and regulation of the CFTR anion channel. Physiol. Rev. 99, 707-738 (2018).

65. Scanlon, K. M. et al. Epithelial anion transporter pendrin contributes to inflammatory lung pathology in mouse models of Bordetella pertussis infection. Infect. Immun. 82, 4212-4221 (2014).

66. Carbonetti, N. H. Bordetella pertussis: New concepts in pathogenesis and treatment. Curr. Opin. Infect. Dis. 29, 287-294 (2016).

67. Borel, J. F., Feurer, C., Gubler, H. U. \& Stähelin, H. Biological effects of cyclosporin A: A new antilymphocytic agent. Agents Actions 6, 468-475 (1976).

68. Liu, J. et al. Calcineurin is a common target of cyclophilin-cyclosporin A and FKBP-FK506 complexes. Cell 66, 807-815 (1991).

69. Fahrer, J. et al. Selective and specific internalization of clostridial C3 ADP-ribosyltransferases into macrophages and monocytes. Cell Microbiol. 12, 233-247 (2010).

70. Ashok, Y. et al. Discovery of compounds inhibiting the ADP-ribosyltransferase activity of pertussis toxin. ACS Infect. Dis. 6, 588-602 (2020).

71. Fulcher, M. L., Gabriel, S., Burns, K. A., Yankaskas, J. R. \& Randell, S. H. Well-differentiated human airway epithelial cell cultures. in Human Cell Culture Protocols 183-206 (Humana Press, Totowa, 2005). https://doi.org/10.1385/1-59259-861-7:183.

72. Neubauer, D. et al. Deuterium oxide dilution: a novel method to study apical water layers and transepithelial water transport. Anal. Chem. 85, 4247-4250 (2013). 


\title{
Acknowledgements
}

This work was supported by the Deutsche Forschungsgemeinschaft [DFG, German Research Foundation, Grant BA2087/2-2 to H.B. and project number 251293561-SFB 1149, Project A05 (H.B. and M.F.)] and the Medical Faculty Ulm (Baustein 3.2 to K.E.). This project is supported by the European Social Fund and by the Ministry of Science, Research and the Arts Baden-Württemberg (fellowship to K.E.). C.K. is supported by a PhD fellowship by Cusanuswerk. V.W. and C.K. are fellows of the International Graduate School in Molecular Medicine Ulm (IGradU) and N.E. and R.L. were supported by the "Promotionsprogramm Experimentelle Medizin" of the Medical Faculty Ulm. Cordelia Schiene-Fischer is thanked for providing Cyp, FKBP and Hsp/c70 proteins, Johannes Buchner for providing Hsp90 protein, and Felix Hausch for FKBP FK1 fragments.

\section{Author contributions}

K.E. designed the study, supervised and conducted experiments and wrote the manuscript. A.K.M., C.K., V.W., N.E., A.A., M.S., R.L. and J.W. conducted experiments. M.S. and A.P. purified recombinant PTxS1 and Gia. C.S. and N.H.C. designed and conducted experiments and wrote parts of the manuscript. M.F. designed experiments and wrote parts of the manuscript. H.B. designed and supervised the study and worked on the manuscript.

\section{Funding}

Open Access funding enabled and organized by Projekt DEAL.

\section{Competing interests}

The authors declare no competing interests.

\section{Additional information}

Supplementary Information The online version contains supplementary material available at https://doi. org/10.1038/s41598-021-84817-2.

Correspondence and requests for materials should be addressed to K.E. or H.B.

Reprints and permissions information is available at www.nature.com/reprints.

Publisher's note Springer Nature remains neutral with regard to jurisdictional claims in published maps and institutional affiliations.

\begin{abstract}
(c) (i)
Open Access This article is licensed under a Creative Commons Attribution 4.0 International License, which permits use, sharing, adaptation, distribution and reproduction in any medium or format, as long as you give appropriate credit to the original author(s) and the source, provide a link to the Creative Commons licence, and indicate if changes were made. The images or other third party material in this article are included in the article's Creative Commons licence, unless indicated otherwise in a credit line to the material. If material is not included in the article's Creative Commons licence and your intended use is not permitted by statutory regulation or exceeds the permitted use, you will need to obtain permission directly from the copyright holder. To view a copy of this licence, visit http://creativecommons.org/licenses/by/4.0/.
\end{abstract}

(C) The Author(s) 2021 